\title{
Advances in thermo-responsive polymers exhibiting upper critical solution temperature (UCST)
}

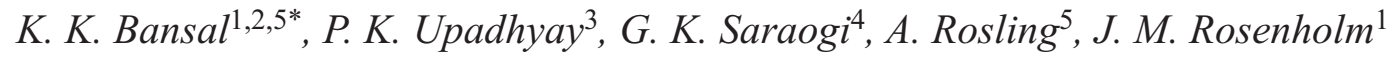 \\ ${ }^{1}$ Pharmaceutical Sciences Laboratory, Faculty of Science and Engineering, Abo Akademi University, 20520 Turku, \\ Finland \\ ${ }^{2}$ Vedica College of Pharmacy, R.K.D.F University, 462033 Bhopal (M.P), India \\ ${ }^{3}$ Institute of Pharmaceutical Research, GLA University, NH-2, Mathura-Delhi Road, 281406 Mathura (U.P.), India \\ ${ }^{4}$ NMIMS School of Pharmacy and Technology Management, 425405 Shirpur (Maharashtra), India \\ ${ }^{5}$ Laboratory of Polymer Technology, Centre of Excellence in Functional Materials at Biological Interfaces, Åbo Akademi \\ University, Biskopsgatan 8, 20500 Turku, Finland
}

Received 1 April 2019; accepted in revised form 9 June 2019

\begin{abstract}
Immense work has been conducted in the field of thermoresponsive polymers specifically of lower critical solution temperature (LCST) type, but upper critical solution temperature (UCST) type polymers remain a significantly unexplored domain. However, in recent years, UCST polymers have attracted increased attention as evidenced by the rise in publications in the same domain, and therefore, this review is an attempt to compile the reported UCST-type polymers. Unlike LCST, UCST polymers are insoluble at low temperature but solubilize in a given solvent as the temperature increases. The synthesis approaches and applications of reported UCST polymers are discussed in this article. Emphasis has been given to the polymers exhibiting UCST behavior in aqueous medium, due to the obvious advantage of their wide applicability. It is quite apparent from this study that the attempts to synthesize novel polymers and copolymers exhibiting UCST has faced an upsurge, but their application part still requires considerable attention.
\end{abstract}

Keywords: smart polymers, thermoresponsive polymers, tailor-made polymers, UCST, nanomaterials

\section{Introduction}

Thermoresponsive or temperature-sensitive polymers are polymers that exhibit reversible sol-gel or gel-sol transition properties in solvent (usually aqueous) above a certain temperature. Such materials are able to undergo conformational changes in response to an internal or external stimuli, and therefore, they are also known as 'stimuli-responsive' or smart polymers [1]. Applications of thermoresponsive polymers have gained immense popularity in several fields including drug delivery, due to the facile control of stimuli-responsiveness. Changes in polymer properties can be stimulated by either introduction of the material into the human body $\left(\sim 35-37^{\circ} \mathrm{C}\right)$ or by applying heat externally in a non-invasive manner. Temperature sensitivity of these polymers can be attributed to the delicate balance between the hydrophilic and hydrophobic components in the structures, and interaction of free functional groups in solvent. Variations in temperature of an aqueous polymer solution/suspension may lead to structural changes in the polymer molecule, which in turn affect its interaction with aqueous medium $[2,3]$. Based on these interactions, polymers have been divided into two categories. The first category of polymers shows phase separation upon heating (Lower Critical Solution temperature (LCST)-type) while, the other one refers to the polymers that show phase separation 
upon cooling (Upper Critical Solution Temperature (UCST)-type) $[4,5]$.

LCST is the temperature below which the polymer is soluble in water, and at temperatures above LCST, the polymer becomes hydrophobic/insoluble [6]. The LCST can be altered as per desired application by fine-tuning the balance between hydrophilic and hydrophobic polymeric components within the macromolecular structure of the co-polymer [7, 8]. On the other hand, UCST is the temperature above which polymeric systems become monophasic or water soluble $[5,9]$. A phase diagram depicting the relationship between concentration of polymer and its solubility as a function of temperature is shown in Figure 1. LCST and UCST are two distinct points in the phase diagram, and represent points of minimum and maximum solubility, respectively, on the solubility curve [10]. At LCST, an order-less arrangement of water molecules causes an increase in entropy of the water in the system, which turns out to be more than the enthalpy observed by water hydrogen attached to the polymer. Therefore, LCST is governed by the system's entropy, whereas UCST is regulated by the enthalpy of the system $[5,8,9,11]$.

Polymers exhibiting UCST and/or LCST are classified as thermoresposive polymers, but polymers showing LCST behavior are dominating in biomedical and similar applications $[2,12]$. The reason for this dissimilarity is linked with the abundant availability of LCST polymers and the obvious advantage of having LCST close to the body temperature. LCST polymers such as poly( $N$-isopropyl acrylamide), poly ( $N, N$-diethylacrylamide), poly $(N$-vinylalkylamide), poly $(N$-vinylcaprolactam), phosphazene derivatives and so forth, have been widely used due to their insolubility at physiological temperature [13-16].

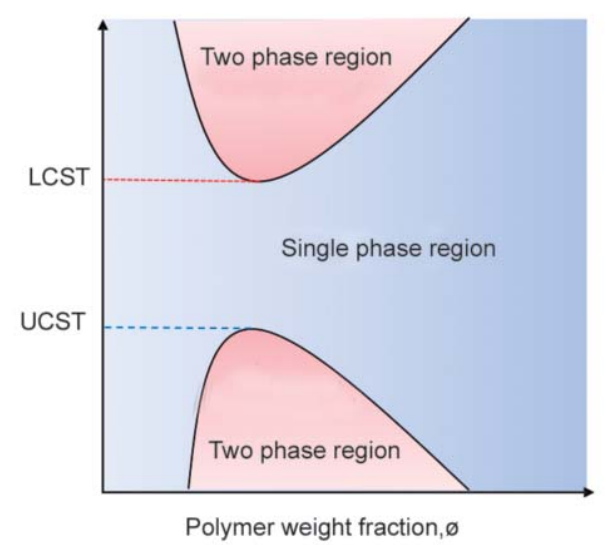

Figure 1. Phase diagram showing LCST and UCST points of a thermoresponsive polymer in aqueous solution.
Nevertheless, Seuring and Agrawal published an excellent review discussing the problems associated with at the time available UCST-based polymers along with the reasons of their ignorance over LCSTbased polymers [9]. Authors clearly gave an indication in this review that although sufficient UCSTtype polymers exist, they are lacking stable UCST behavior and therefore, guidelines are provided to aid researchers in designing optimum polymers. UCST of these polymers are affected by $\mathrm{pH}$, molecular weight, ionic strength, presence of electrolytes, and polymer concentration in solution. In general, UCST polymers exhibit thermoresponsive behavior on the basis of cohesive interactions, such as hydrogen bonding or electrostatic interactions between polymer chains, that are destabilized at higher temperature $[9,17]$.

Zwitterionic polymers (e.g. poly(betaines)) that demonstrate UCST based on electrostatic interactions, are very sensitive to the presence of electrolytes and concentration $[9,18]$. However, it has been observed that certain novel non-ionic polymers are very tolerant to the presence of electrolytes and exhibit sharp UCST transitions over a wide spectrum of concentration of electrolytes. The tendency of a non-ionic polymer to exhibit UCST behavior is to a great extent governed by the ability of the polymer to form hydrogen bonds with molecules of water in the media, especially through functional groups such as primary amide. With increase in temperature, the hydrogen bonds between polymer side groups are broken (endothermic process) and replaced by hydrogen bonds with water molecules (exothermic process) [8]. Poor strength of these inter- and/or intramolecular bonds are responsible for demonstrating UCST behavior of such polymers [10]. Thus, ionic strength of a medium does not influence the phase transition for hydrogen bonding based UCST polymers, and therefore, they are promising alternatives for biomedical applications [19, 20]. Furthermore, the UCST of polymers can easily be varied based on the required application, and the existing methodologies have been recently reviewed [8].

The aim of this review is to bring up-to-date information to the readers regarding the advancements within the field of UCST-type polymers. Synthesis approaches utilized to prepare novel polymers or copolymers exhibiting UCST in aqueous medium have been discussed. Further, possible applications 
of these UCST-type polymers based on the published literature have been reviewed.

\section{Polymers exhibiting upper critical solution temperature}

\subsection{Poly(sulfobetaine)}

Polymer based on sulfobetaines are zwitterionic polymers usually synthesized via radical/ reversible addition and fragmentation chain transfer (RAFT) polymerization of sulfobetaine monomers [21-23]. Thermoresponsive derivatives of poly(sulfobetaine) displayed UCST behavior via electrostatic interaction in aqueous medium, and therefore their UCST largely depends on salt concentration owing to the presence of charged ammonium and sulfonate groups in the chemical structure [18, 24]. Reduction of UCST in the presence of salts limits their applications, and therefore Woodfield et al. [25] prepared hydrophobically modified poly(sulfobetaine) to enhance the aqueous UCST (Figure 2). Despite the reports suggesting increase in UCST by addition of hydrophobic moiety into the homopolymer [8], the authors observed a decrease in UCST of pentyl modified copolymer (Figure $2 b$ ) compared to homopolymer (UCST $\sim 8.5^{\circ} \mathrm{C}$ at a concentration of $10 \mathrm{~g} / \mathrm{l}$ ). However, addition of benzyl groups leads in UCST surge from 14.5 to $82.4^{\circ} \mathrm{C}$, whereas substitution of dodecylamine makes the polymer insoluble [25]. Similarly, Roth and coworkers synthesized sulfobutylbetaine with tunable UCST via three different synthetic routes. Quaternization of poly[2-(dimethylamino)ethyl methacrylate] by postpolymerization with 1,4-butanesultone, RAFT polymerization of sulfobutylbetaine-functional (meth)acrylate monomers and their copolymerization with a sulfopropylbetaine (SPB) methacrylate and modification of poly(pentafluorophenyl acrylate) (pPFPA) by post-polymerization with sulfobutylbetaine -functional amine and with benzylamine as a hydrophobic modifier were the chosen strategies. The authors demonstrated that critical solution temperatures of homopolymers can be increased simply by increasing the ion bridge by one methylene unit [22]. An increment in UCST of poly(sulfobetaine methacrylates) was also reported by increasing the spacer length separating the ammonium and the sulfonate groups [26].

Copolymerization of sulfobetaine methacrylic-based monomer [2-(methacryloyloxy)ethyl]dimethyl-(3sulfopropyl)ammonium hydroxide (SBE) and carboxybetaine methacrylic-based monomer 2-((3methacrylamidopropyl)dimethylammonio)acetate (CBA) is an another approach used to increase the UCST of sulfobetaine based polymers, and simultaneously to insert the free carboxyl functional groups in copolymer for post-modification (Figure 3) [27]. Recently, the synthesis of a double zwitterion block copolymer i.e. poly(2-((2-(methacryloyloxy)ethyl) dimethylammonio)acetate)- $b$-poly(3-( $N$-(2-metharyloylethyl)- $N, N$-dimethylammonio)propanesulfonate) (PGLBT- $b$-PSPE) was reported using two different polybetaine blocks. Both PGLBT and PSPE are hydrophilic, but PSPE demonstrate UCST-behavior
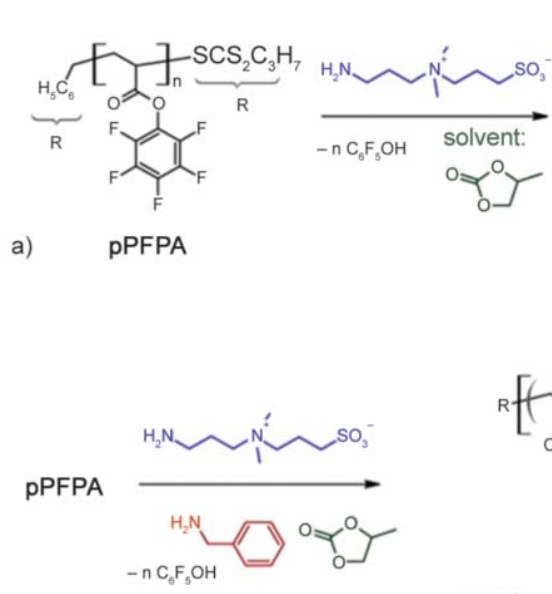

c)

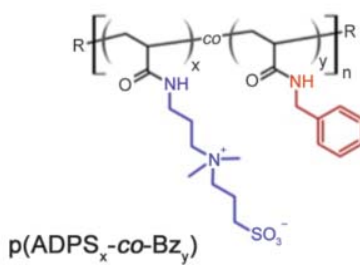

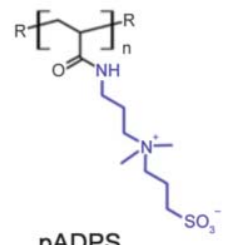

pADPS

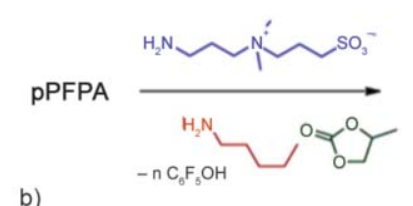

b)

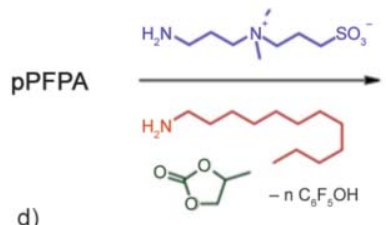

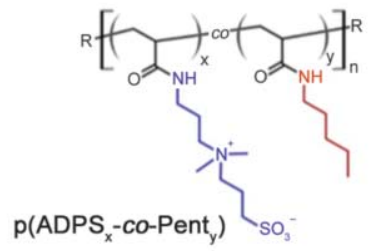

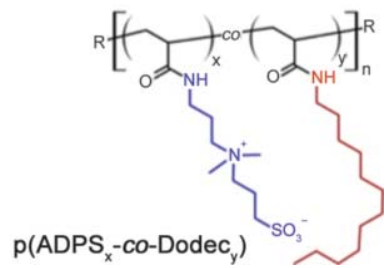

Figure 2. Synthesis scheme of sulfobetaine copolymers derivatized by difference hydrophobic comonomers (a) synthesis of homopolymer poly[(3-((3-acrylamidopropyl)dimethylammonio)propane-1-sulfonate] (pADPS) using poly(pentafluorophenyl acrylate (pPFPA) as precursor (b) pentylamine modified sulfobetaine copolymer (c) benzylamine modified sulfobetaine copolymer and (d) dodecylamine modified sulfobetaine copolymer using propylene carbonate as solvent. Reproduced with permission from [25], Copyright (C) 2014 American Chemical Society. 


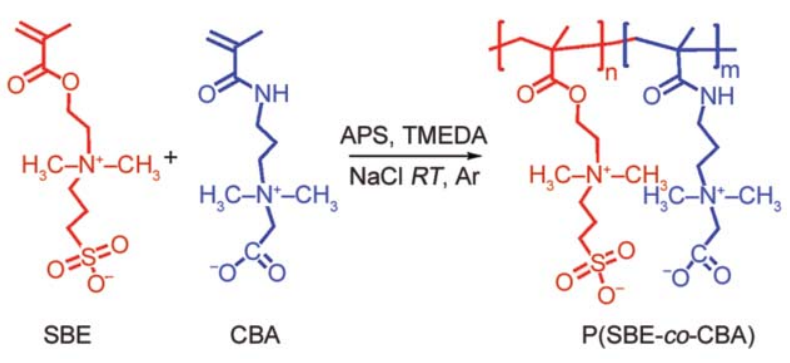

Figure 3. Synthesis scheme of [2-(methacryloyloxy)ethyl] dimethyl-(3-sulfopropyl)ammonium hydroxide (SBE) and carboxybetaine methacrylic-based monomer 2-((3-methacrylamidopropyl)dimethylammonio)acetate (CBA) copolymerization using $N, N, N^{\prime}, N^{\prime}$-tetramethylethylenediamine (TMEDA) as initiator ( $R T$ - room temperature, APS - ammonium peroxodisulfate, $\mathrm{Ar}$ - argon, $\mathrm{NaCl}$ - sodium chloride). Reproduced with permission from [27], Copyright (C) 2012 American Chemical Society.

and become less soluble in water below UCST (UCST $\sim 45^{\circ} \mathrm{C}$ ). Hence, PGLBT- $b$-PSPE formed micelles with PSPE core and PGLBT shell below UCST but this assembly disrupt upon heating above UCST [23]. Similarly, micelle formation and temperature induced disassembly of block copolymers containing derivative of poly(sulfobetaine) as one of the block were reported [28-30]. There are several other reports presenting the synthesis of thermoresponsive derivatives of poly(sulfobetaine) with tunable UCST, however, the application part of such polymers were sparsely explored due to the high fluctuations of UCST in the presence of electrolytes.

\subsection{Poly(acrylamide-co-acrylonitrile) poly(AAm-co-AN)}

Poly(acrylamide) is a nonionic water-soluble polymer that features a primary amide group with wellknown long history of its use in cosmetic products and as a hydrogel [31, 32]. However, it does not exhibit thermo-responsive behavior on its own (theoretical UCST $<0^{\circ} \mathrm{C}$ ). Considering the advantages such as capability of poly(acrylamide) to form reversible hydrogen bonding and low content of ionic groups, Seuring and Agarwal copolymerized acrylamide (AAm) and acrylonitrile (AN) to generate a series of nonionic thermoresponsive copolymers, whose UCST were less sensitive to electrolytes. The polymers were synthesized by keeping constant AAm concentration via free radical polymerization, using 2,2'Azoisobutyronitrile (AIBN) as initiator containing varying amounts of $\mathrm{AN}$. It was proposed that by changing the molar ratio of hydrophobic AN in Poly(AAm-co-AN), the UCST of polymer can be tuned easily (Figure 4) [33]. Later, Asadujjaman et al. [34] suggested that Poly(AAm-co-AN) with AN content between 0.086 to 0.221 only demonstrate UCST behavior with cloud point ranging from 5.5 to $56.5^{\circ} \mathrm{C}$ at $1 \mathrm{mg} / \mathrm{ml}$ concentration in water. This report suggested that the phase transition temperature $\left(T_{\mathrm{pt}}\right)$ increases with increase in AN content in the copolymer, increasing polymer concentration and polymer chain length and by addition of $300 \mathrm{mM} \mathrm{Na}_{2} \mathrm{SO}_{4}$ in solution. It should be noted that the $T_{\mathrm{pt}}$ of $\mathrm{P}(\mathrm{AAm}-\mathrm{co}-\mathrm{AN})$ in $\mathrm{H}_{2} \mathrm{O}$ is observed to be almost $10^{\circ} \mathrm{C}$ lower than in $\mathrm{D}_{2} \mathrm{O}$ at constant concentration. The reason behind this solvent isotope effect is attributed to the formation of inter-/intra-chain hydrogen bonds in $\mathrm{D}_{2} \mathrm{O}$ by all $\mathrm{C}=\mathrm{O}$ groups compared to $\mathrm{H}_{2} \mathrm{O}$ solution where a part of $\mathrm{C} \mathrm{O}$ groups are free from $\mathrm{C}=\mathrm{O} \cdots \mathrm{H}-\mathrm{N}$. In addition, it has been found that addition of $150 \mathrm{mM} \mathrm{NaCl}$ decreases $T_{\mathrm{pt}}$ of P(AAmco-AN) by $5^{\circ} \mathrm{C}[35]$.

Similarly, bile acid conjugated acrylamide monomer was copolymerized with acrylamide via RAFT polymerization to generate UCST polymer analogous to Poly(AAm-co-AN), in which AN was replaced with

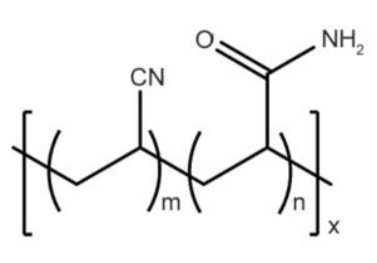

a)

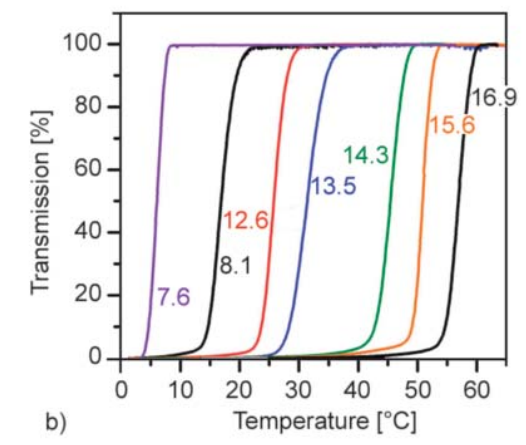

Figure 4. (a) Representative structure of poly(acrylamide-co-acrylonitrile) and (b) turbidity cooling curves of poly(AAmco-AN) with different acrylonitrile content in mole\% (numbers in graph). Reproduced with permission from [33], Copyright (C) 2012 American Chemical Society. 

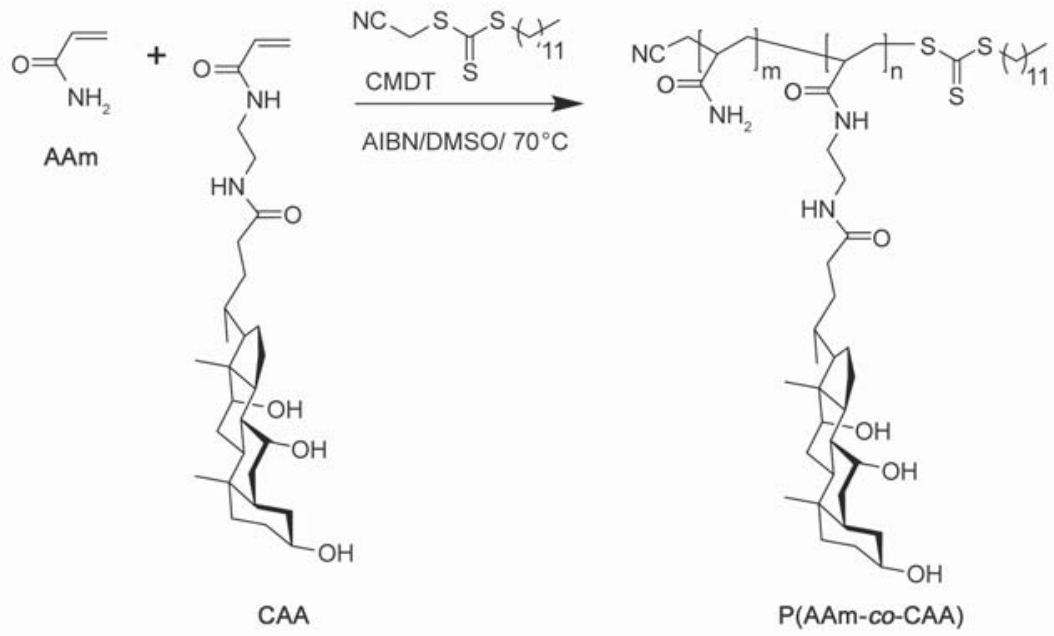

Figure 5. Synthesis scheme of UCST polymer P(AAM-co-CAA) via RAFT polymerization using of cholic acid conjugated acrylamide (CAA) and acrylamide (AAm) as monomers in DMSO. (CMDT - cyanomethyl dodecyl trithiocarbonate, AIBN -2,2'-Azoisobutyronitrile). Reproduced with permission from [36], Copyright (C 2017 American Chemical Society.

water insoluble acrylamide containing bile acid moieties (Figure 5). Tpt of this copolymer can be easily tuned between 5 to $85^{\circ} \mathrm{C}$ by varying the molar fraction of bile acid monomer in the copolymers. Further, authors also demonstrate that the UCST of copolymer could also be controlled by using $\beta$-cyclodextrin ( $\beta-C D)$ as an additive in water [36]. Käfer et al. [4] utilized poly(ethylene glycol) (PEG, $M_{\mathrm{n}}$ $2000 \mathrm{~g} / \mathrm{mol}$ ) as a macro-azoinitiator to impart dual thermoresponsive property in $\mathrm{P}(\mathrm{AAm}-\mathrm{co}-\mathrm{AN})$ copolymer. This new diblock copolymer PEG- $b$-(AAmco-AN) with 9 mole $\%$ of AN demonstrate LCST at $\sim 30^{\circ} \mathrm{C}$ and UCST at $\sim 50^{\circ} \mathrm{C}$. The Tpt of this diblock

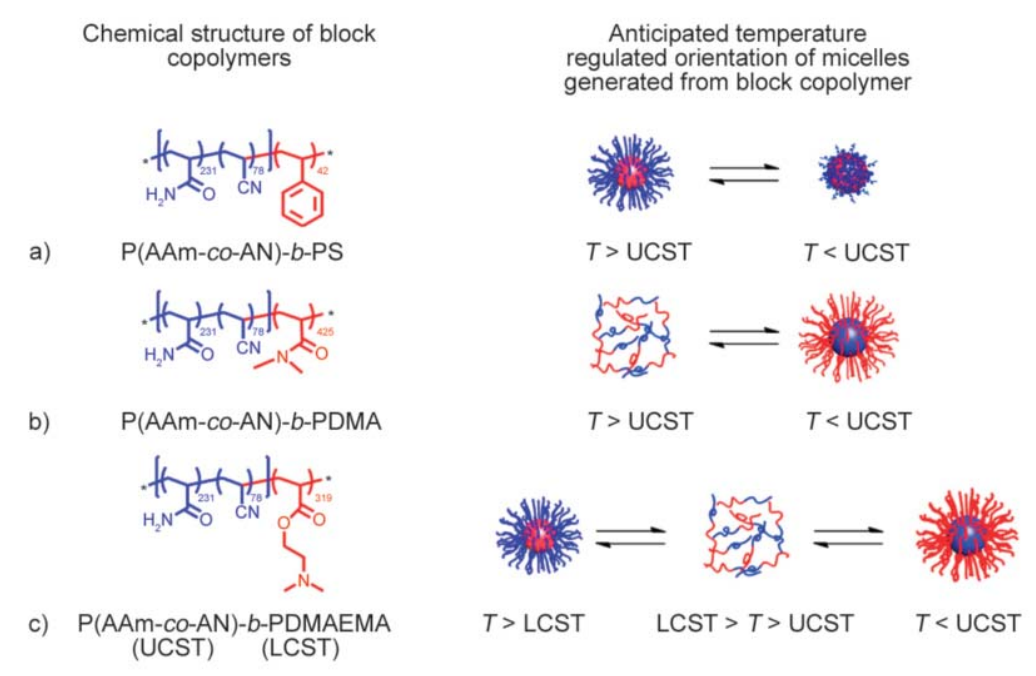

Figure 6. Illustration of chemical structure of block copolymers and their corresponding self-assembly in micelles and their anticipated conformation change with change in temperature. (a) Poly(acrylamide-co-acrylonitrile)-blockpoly(styrene) (b) poly(acrylamide-co-acrylonitrile)-block-poly(dimethylacrylamide), (c) poly(acrylamide-co-acrylonitrile)-block-poly( $N, N$-dimethylaminoethyl methacrylate). Reproduced with permission from [37], Copyright (C) 2014 American Chemical Society.

copolymer was found to be dependent on the PEG block length, AN content, and the polymer concentration in solution. The cytotoxicity study on L929 cell lines suggested that addition of PEG to P(AAmco-AN) copolymer reduced the toxicity up to 1.5 fold compared to unmodified P(AAm-co-AN). Block copolymer of P(AAm-co-AN) with hydrophobic polystyrene or hydrophilic poly(dimethylacrylamide) or with LCST polymer poly( $N, N$-dimethylaminoethyl methacrylate) has been also reported with the objective to alter UCST and orientation of assembly. These block copolymer assembled in micelles when dispersed in water or phosphate-buffered 
saline and demonstrate UCST-based changes such as reversible dispersion-aggregation of micelles, dissolution-formation of micelles, and reversal of micelle core and corona respectively (Figure 6) [37]. Copolymerization of $\mathrm{P}(\mathrm{AAm}-\mathrm{co}-\mathrm{AN})$ with PVP was also explored in order to generate a LbL temperature-responsive films, which assembled in well-defined block copolymer micelles below UCST [38]. Qi et al. [39] reported synthesis of first hyperbranched UCST polymer using poly[3-ethyl-3-(hydroxymethyl) oxetane] (HBPO) as core and $\mathrm{P}(\mathrm{AAm}-\mathrm{co}$ AN) as arm. Hydroxyl end groups of HBPO was first converted into carboxylic acid by attaching maleic anhydride followed by generation of RAFT agent HBPO-DTBA (poly[3-ethyl-3-(hydroxymethyl)oxetane]-dithiobenzoic acid). RAFT polymerization was then performed using Aam and AN as monomer, AIBN as initiator and HBPO-DTBA as RAFT agent (Figure 7). In contrast to the report of Asadujjaman et al. [34], the UCST of this novel hyperbranched polymer can be increased by decreasing the arm (chain) length due to its unique molecular topology [39]. Synthesis of an ABA type triblock copolymers using $\mathrm{P}(\mathrm{AAm}-\mathrm{co}-\mathrm{AN})$ as outer blocks or middle block and hydrophilic poly(poly(ethylene glycol) methyl ether methacrylate) (PPEGMMA) and poly ( $N, N$-dimethylacrylamide-co-7-acryloyl-4-methylcoumarin) as middle block and outer block, respectively, have also been reported [40, 41]. This triblock copolymer exhibiting temperature induced reversible sol-gel transitions, and therefore its applications in sustained release and as injectable biomedical scaffold has been proposed.

\subsection{Poly( $N$-acryloylglycinamide)}

Poly( $N$-acryloyl glycinamide) (PNAGA) (Figure 8) synthesized by free radical polymerization from vinyl monomer $N$-acryloyl glycinamide (NAGA) is an another example of non-ionic polymer exhibiting UCST behavior [42, 43]. Although PNAGA has been known since 1964, its UCST was only recently reported by Seuring and Agarwal; which is $21.5 \pm 2.2{ }^{\circ} \mathrm{C}$ upon
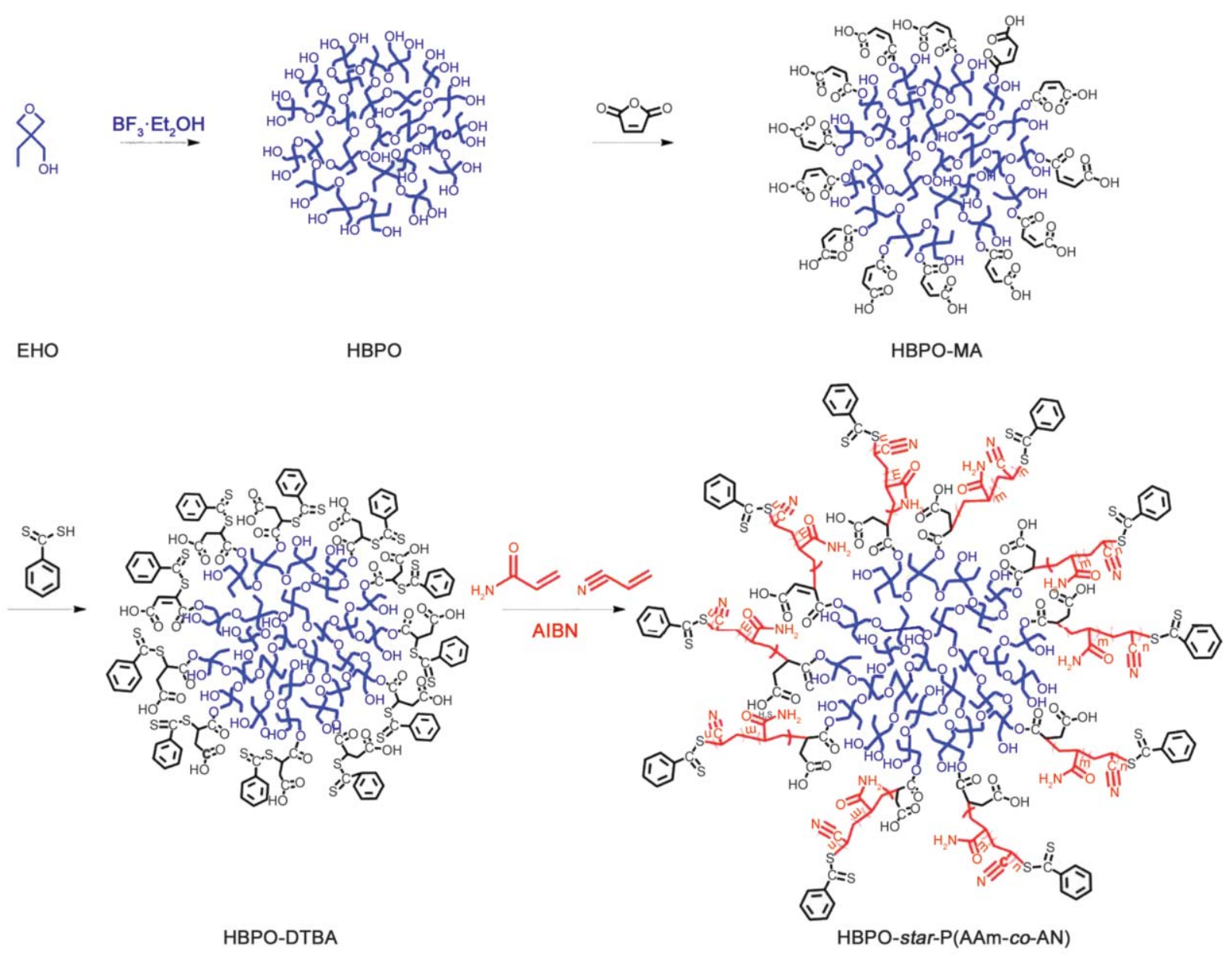

Figure 7. Step-by-step synthesis of HBPO-star-P(AAm-co-AN). (EHO-[3-ethyl-3-(hydroxymethyl)oxetane], monomer for synthesis of hyperbranched HBPO. Reproduced with permission from [39], Copyright (C 2018 American Chemical Society. 
<smiles>C=CC(=O)NCC(N)=O</smiles>

$\mathrm{N}$-acryloyl glycinamide

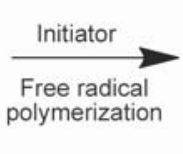

Figure 8. Illustration of poly( $N$-acryloyl glycinamide) (PNAGA) synthesis.

heating and $12.3 \pm 0.3{ }^{\circ} \mathrm{C}$ upon cooling in water [10]. Later, Agarwal and coworker suggested that traces of ionic groups present in poly(NAGA) were most likely responsible for the absence or irreproducible UCST behavior. To confirm this speculation, acrylate free starting material NAGA was synthesized and utilized for PNAGA synthesis. It was suggested that the presence of potassium acrylate in the monomer can be converted into acrylic acid upon polymerization and restrain the UCST behavior of poly(NAGA). As a result, PNAGA synthesized using acrylate free NAGA exhibit UCST while polymer prepared using conventionally synthesized NAGA did not show UCST. Hydrolysis of the amide group of PNAGA and use of ionic initiators in radical polymerization are other factors, which contribute in suppressing the UCST behavior of polymer [42].

However, it has been observed that polymer concentration in solution affects the UCST, and therefore Käfer et al. [44] synthesized a copolymer of PNAGA using AN as second block (Figure 9) to avoid the effect of concentration and to retain narrow cooling/ heating hysteresis in water. The $T_{\mathrm{pt}}$ of copolymer $\mathrm{P}(\mathrm{NAGA}-\mathrm{co}-\mathrm{AN})$ was found to be independent of polymer concentration, and can be easily tuned by altering the AN content in copolymer. This copolymer self-assembled into micelle-like structures above UCST, owing to the insolubility of AN while formed

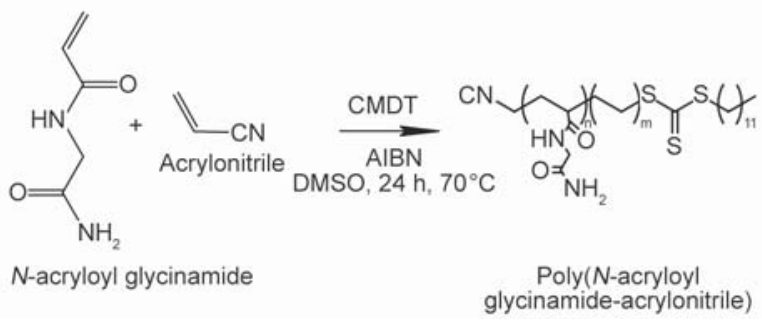

Figure 9. Copolymerization of $N$-acryloyl glycinamide and acrylonitrile by using cyanomethyl dodecyl trithiocarbonate (CMDT) as chain transfer agent and 2,2'-Azoisobutyronitrile (AIBN) as initiator [44].

aggregates below UCST as evident by transmission electron microscopy images. Sun et al. [45] reported synthesis of a dual thermoresponsive copolymer i.e. $\mathrm{P}(\mathrm{NAGA}-\mathrm{co}$-DAAM) using NAGA and diacetone acrylamide (DAAM) via RAFT polymerization, which demonstrated both UCST and LCST type transitions (Figure 10). The $T_{\mathrm{pt}}$ are tunable by changing composition of NAGA and DAAM, molecular weight of polymer, polymer concentration, isotope effect and the presence of electrolyte in media. By increasing the molar fraction of DAAM, a decrease in $T_{\mathrm{pt}}$ with narrow hysteresis was observed for UCSTtype copolymers. Similarly, copolymerization of NAGA with methacrylic acid (MA) was executed to tune the UCST of polymer near to human body temperature. The resultant copolymer P(NAGA-co-MA) demonstrate UCST between 17 to $37^{\circ} \mathrm{C}$ based on the

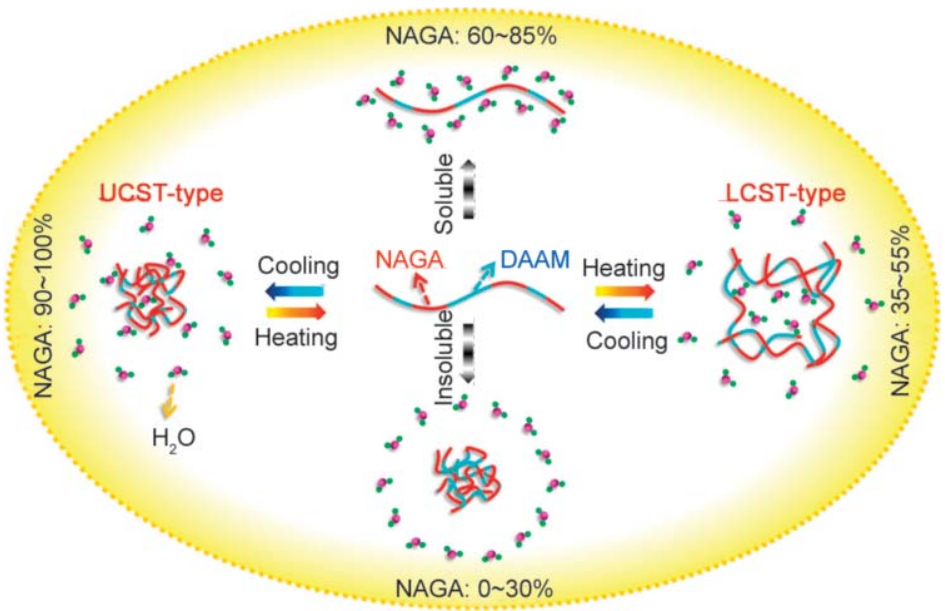

Figure 10. Graphical presentation of UCST and LCST type behavior of P(NAGA-co-DAAM) copolymers. Reproduced with permission from [45], Copyright (C) 2017 American Chemical Society. 
molar fraction of MA, which was in the range of 30 $60 \mathrm{~mol} \%$. However, the UCST of P(NAGA-co-MA) is dependent on $\mathrm{pH}$, ionic strength and polymer concentration, thus limiting its broad applicability [46].

\subsection{Ureido-derivatized polymers}

The ureido group has a high number of $\mathrm{H}$-bonding sites and thus, it is expected that this group could readily form hydrogen bonding, which is essential for non-ionic polymers to demonstrate UCST behavior. Therefore, Shimada et al. [47] synthesized a copolymer poly(allylamine)-co-poly(allylurea)s (PAUs) containing ureido groups to impart UCST behavior (Figure 11) . PAUs demonstrate UCST with high stability in solution and the $T_{\mathrm{pt}}$ was found to increase linearly with increase in ureido content, which can be tuned between 8 to $65^{\circ} \mathrm{C}$ under physiological salt and $\mathrm{pH}$ conditions. To validate the importance of ureido groups in producing UCST-type behavior, polypeptides poly(L-ornithine)-co-poly(L-citrulline)s

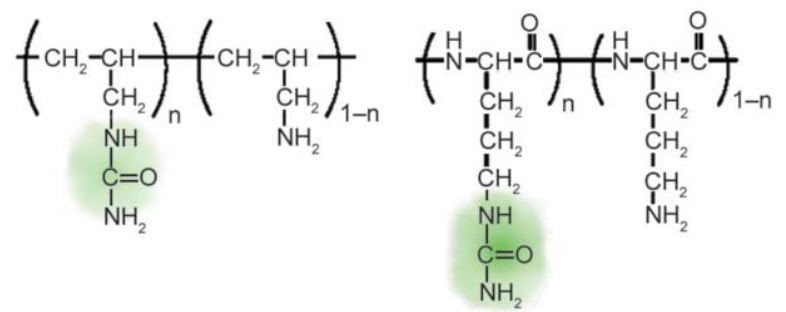

Poly(allylamine)-co-poly(allylurea) Poly(L-ornithine)-co-poly(L-citrulline)

Figure 11. Structural formula of poly(allylamine)-co-poly (allylurea) and poly(L-ornithine)-co-poly(L-citrulline) containing ureido groups (highlighted in green colour). Reproduced with permission from [47], Copyright (C) 2011 American Chemical Society.
(POCs), containing ureido groups were prepared. As expected, POCs noticeably demonstrated UCST behavior under physiological conditions, which was dependent on both ureido content and molecular weight of main chain. Later, Maruyama and coworkers developed hydrophobically modified poly(allylamine)-co-poly(allylurea)s by acylation of amino groups of poly(allylamine) in order to reduce the influence of $\mathrm{pH}$ on $T_{\mathrm{pt}}$, and also suggested that stereoregularity of the polypeptides has a significant effect on UCST behavior [48, 49].

Palanisamy et al. [50] reported synthesis of an amphiphilic type of copolymer using UCST polymer polyureido(ornithine-co-lysine) and hydrophilic polyvinylpyrrolidone (PVP) in order to generate polymer possessing self-assembly behavior in aqueous medium. UCST of this copolymer can be tuned between $14-33^{\circ} \mathrm{C}$ by changing the ornithine-to-lysine ratio and by molecular weight. However, it was observed that the UCST behavior of assembly and disassembly of micelles at $33^{\circ} \mathrm{C}$ was not reproducible. Nevertheless, these micelles can be stabilized by depositing within layer-by-layer (LbL) films via hydrogen bonding with tannic acid (TA). Synthesis of hyperbranched, star-like polypeptides, SPOC, has also been reported via ring-opening polymerization (ROP) using fourth generation polyamidoamine dendrimer (G4-PAMAM) as an initiator and carboxybenzyl protected L-ornithine $N$-carboxyanhydride (NCA) as monomer. After deprotection of carboxybenzyl groups of NCA, pendant primary amine groups of the star polymer were substituted with ureido groups to insert UCST-type property (Figure 12). As demonstrated

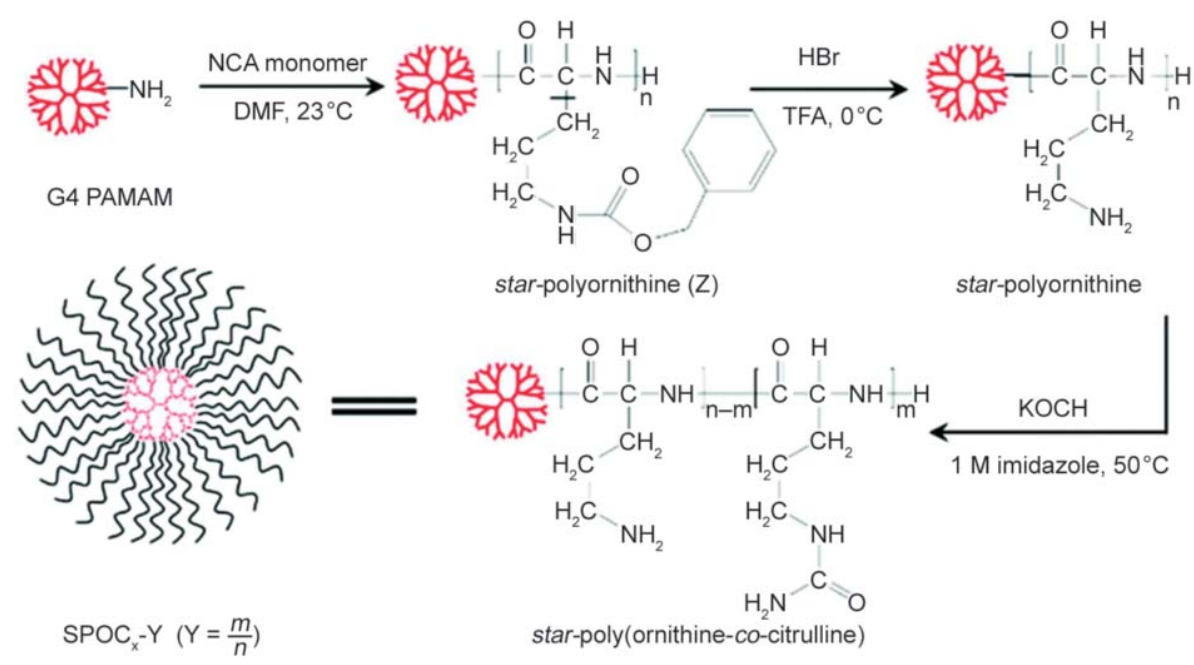

Figure 12. Synthesis scheme for dendritic SPOC polymers using G4-PAMAM dendrimer as initiator (core) and protected NCA as monomer via ROP (KOCN - potassium cyanate). Reproduced with permission from [51], Copyright (C) 2018, The Royal Society of Chemistry. 
previously, the UCST of this enzymatically degradable star polymer is tunable through degree of ureido modification and by change length of polypeptide arms [51].

Ureido groups containing polymer i.e. poly(2-ureidoethylmethacrylate) (PUEM) have also been synthesized by modifying the amine groups of poly(2aminoethyl methacrylate hydrochloride) (Figure 13). The $T_{\mathrm{pt}}$ of these polymers in PBS can be tuned by changing molecular weight of the polymer, polymer concentration, and $\mathrm{NaCl}$ concentration $[52,53]$. A

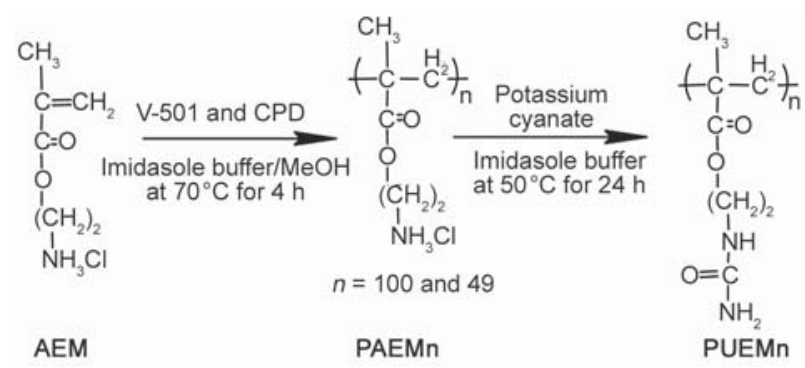

Figure 13. Synthetic route of poly(2-ureidoethylmethacrylate) using starting material 2-Aminoethyl methacrylate hydrochloride (AEM) (V-501-4,4'azobis(4-cyanovaleric acid), CPD - 4-Cyanopentanoic acid dithiobenzoate). Reproduced with permission from [52], Copyright (C) 2016 Wiley Periodicals, Inc.

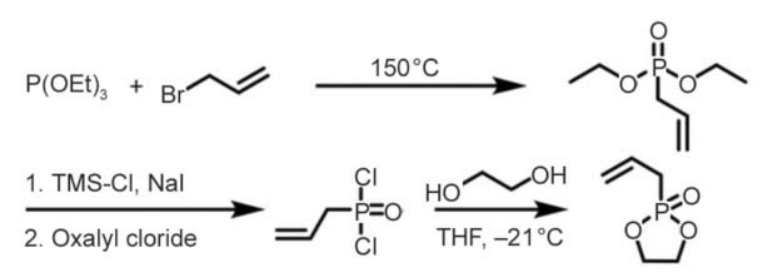

a)

(2)

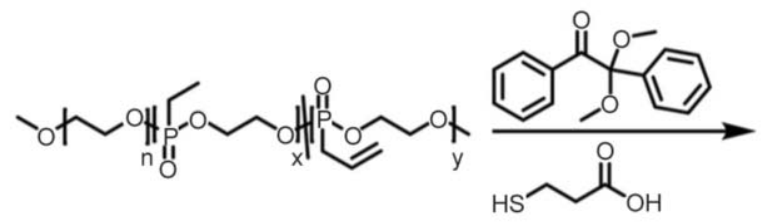

c)

$$
P\left(1_{x}-c o-2\right)
$$

copolymer of PUEM with hydrophilic poly(2methacryloyloxyethyl phosphorylcholine) (PMPC) has been synthesized to demonstrate the temperature facilitated self-assembly in micelles below UCST. It was suggested that this polymeric micelles with biocompatible PMPC shells could be used as drug delivery carrier for designing temperature responsive formulations, since these polymeric micelles can incorporate hydrophobic guest in PUEM core below UCST and could offer temperature-guided controlledrelease of loaded cargo [53].

\subsection{Other polymers}

The majority of polymers showing UCST behavior are non-biodegradable and, therefore, to overcome this limitation, Wolf et al. [54] reported synthesis of degradable poly(ethylene alkyl phosphonate) with adjustable UCST. Water-soluble copolymer was synthesized via anionic ring opening polymerization using 2-ethyl-2-oxo-1,3,2-dioxaphospholane and 2allyl-2-oxo-1,3,2-dioxaphospholane as monomer and methoxy-PEG as initiator (Figure 14a and 14b). Later, 3-mercaptopropionic acid was attached to pendent double bond via thiol-ene click reaction to introduce hydrophobicity and H-bonding capability to the

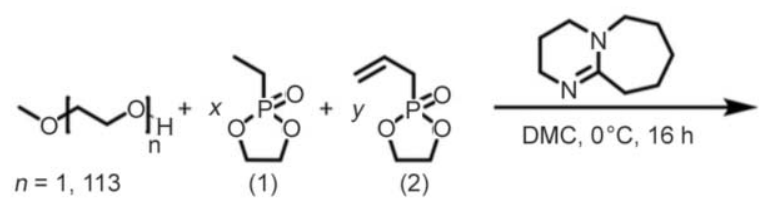

b)

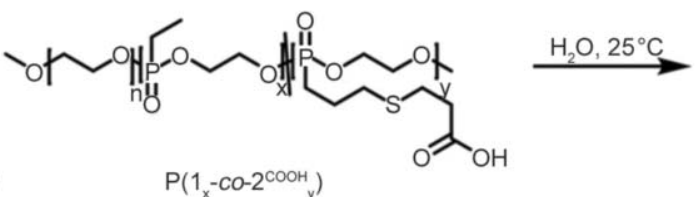

e)

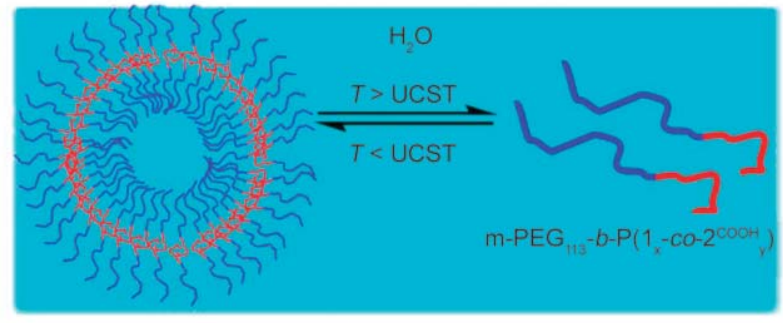

Figure 14. (a) Synthesis of monomer 2-allyl-2-oxo-1,3,2-dioxaphospholane (2), (b) anionic ring opening polymerization of 2-ethyl-2-oxo-1,3,2-dioxaphospholane (1) and (2) using DBU as a catalyst and methoxy-PEG as initiator to produce copolymer $\mathrm{P}\left(1_{\mathrm{x}}-c o-2 \mathrm{y}\right)$, (c) attachment of 3-mercaptopropionic acid via thiol-ene click reaction onto $\mathrm{P}\left(1_{\mathrm{x}}-\right.$ $c o-2$ ) to generate (d) $\mathrm{P}\left(1_{\mathrm{x}}-\mathrm{CO}-2^{\mathrm{COOH}}\right)$, and (e) representation of assembly and disassembly of polymer above and below UCST, Reproduced with permission from [54], Copyright (C) 2017 American Chemical Society. 
polymer (Figure 14c and 14d). This copolymer demonstrate UCST behavior in water and self-assemble in polymerosomes below UCST due to the precipitation of poly(ethylene alkyl phosphonate) block. In contrast, structural disassembly has been observed at temperatures above UCST, due to increase in solubility of poly(ethylene alkyl phosphonate) block in water (Figure 14e). The UCST of this copolymer can be tuned by varying the amount of mercaptopropionic acid (i.e. $-\mathrm{COOH}$ groups) in feed during thiolene click reaction [54]. However, the UCST of synthesized copolymer was dependent on $\mathrm{pH}$, electrolyte and polymer concentration, which might restrict the obvious advantage of degradability associated with this polymer in biomedical applications. Di et al. [55] reported the synthesis of a novel kind of thermosensitive polymer poly $(N$-propionyl-aspartic acid/ethylene glycol) via polycondensation reaction using L-aspartic acid and ethylene glycol as monomers. This UCST polymer demonstrated no cytotoxicity when tested on HeLa cells for $48 \mathrm{~h}$. It was proposed that the thermosensitive behavior with tunable UCST (between $1.5-37.6^{\circ} \mathrm{C}$ by varying alcohol or $\mathrm{NaCl}$ content in water) along with absence of cytotoxicity could be the advantage of this novel polymer to find applications in the biomedical field. In an another example, biodegradable polyglycerol sebacate (PGS) based copolymers were prepared by modifying PGS hydroxyl handles with polyethylene glycol methyl ether methacrylate (PEGMEMA) to yield water-soluble PGS-PEGMEMA copolymer. Addition of this copolymer with $\alpha$-cyclodextrin (CD), results in a supramolecular hydrogel system possessing UCST behavior, which could be easily tuned by varying the $\alpha$-CD concentration. Due to the advantages such as rapid gelation, low minimum gelation concentration, and rapid self-healing ability, it was proposed that this hydrogel system could find applications in biomedical field [56].

Wu et al. [57] reported synthesis of a biodegradable and biocompatible UCST-type copolymer i.e. $\operatorname{poly}(p-$ dioxanone)-grafted poly(vinyl alcohol) (PVA-gPPDO). This copolymer demonstrate the ability to switch between LCST-type and UCST-type thermoresponsivity by adjusting the chain length of PPDO block. A similar work was reported by Zhang et al. [58], in which synthesis of novel copolymer poly (imidazoled glycidyl methacrylate-co-diethylene glycol methyl ether methacrylate) (P(iGMA-co- $\left.\mathrm{MEO}_{2} \mathrm{MA}\right)$ ), exhibiting both LCST-type and UCST-type thermoresponsivity was discussed. The copolymer was prepared in two steps in which the first step involve synthesis of copolymer poly(glycidyl methacrylate-codiethylene glycol methyl ether methacrylate) via atom transfer radical polymerization (ATRP) followed by opening of epoxide rings of poly (glycidyl methacrylate) by reacting with imidazole groups to generate $\mathrm{P}\left(\mathrm{iGMA}-\mathrm{co}-\mathrm{MEO}_{2} \mathrm{MA}\right)$. It is worth noting that the homopolymers of $\mathrm{MEO}_{2} \mathrm{MA}$ and iGMA display LCST and UCST behavior, respectively, and therefore the LCST to UCST transition of copolymer can be achieved by changing the molar ratio of iGMA to $\mathrm{MEO}_{2} \mathrm{MA}$.

\section{Applications of UCST-type polymers \\ 3.1. Energy storage device}

Polymers that show UCST in polar organic systems have been utilized to design energy storage devices, which could be suitable for higher voltage applications such as supercapacitors and Li-ion batteries. A temperature sensitive ion permeable membrane has been designed by coating UCST polymer poly(sulfobetaine) on graphene oxide sheets. It was suggested that at temperatures below UCST, the dipolar attraction between polymer zwitterions in organic electrolyte leads to polymer coiling. Consequently, aggregates were formed onto the membrane and thus, this provided an uninterrupted ion flux through open channels available in the membrane. In contrast, at temperatures above UCST the dipolar attraction impeded, which caused uncoiling of polymer chains and unveiling zwitterions to the electrolyte, and ultimately, lowering the ion permeation through the membrane. Therefore, this membrane was expected to be useful in alleviating thermal runaway in batteries by reducing ion mobility at higher temperature, thereby decreasing the charge-discharge rates and heat generation [59].

\subsection{Controlled drug release}

A series of UCST polymer mPEG- $b$-P(AAm-coAN) was utilized for controlled release of doxorubicin (DOX), which was regulated by temperature $[60,61]$. An amphiphilic polymer containing $9.13 \%$ of acrylonitrile exhibit UCST of $43.1^{\circ} \mathrm{C}(0.3 \mathrm{mg} / \mathrm{ml}$ concentration) was capable of forming micelles with a CMC of $23.64 \mu \mathrm{g} / \mathrm{ml}$. The in-vitro drug release study at a temperature below UCST $\left(4^{\circ} \mathrm{C}\right)$, near 
$\operatorname{UCST}\left(42^{\circ} \mathrm{C}\right)$ and above UCST $\left(70^{\circ} \mathrm{C}\right)$ suggested acceleration in release rate as the temperature increases (Figure 15). However, the micelle disassembly and complete release of drug at temperatures above UCST was not observed as expected. The reason behind this phenomenon was attributed to a change in UCST after DOX loading. The authors also reported decrease in UCST at lower concentrations of polymer, which could hinder the biomedical use of this system, as the formulation is subjected to dilution in blood after IV administration [61]. Therefore, additional

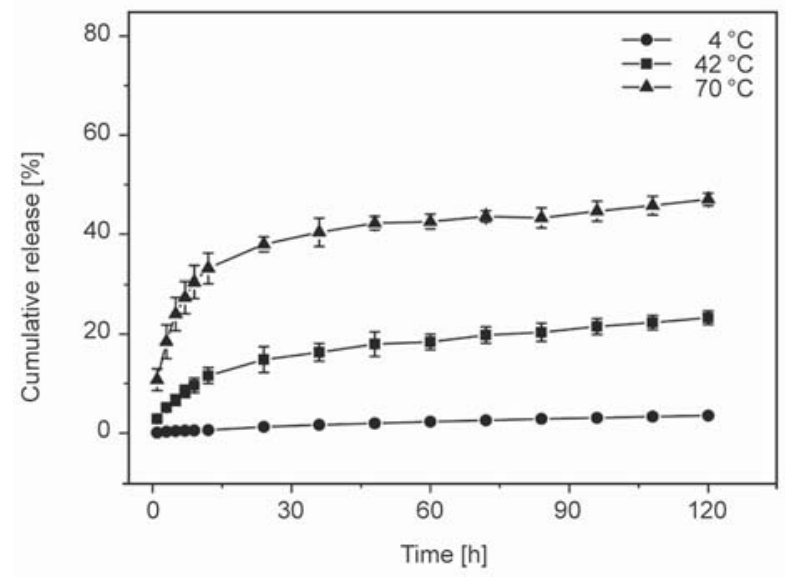

Figure 15. Cumulative release of doxorubicin from $\mathrm{mPEG}-$ $b$-P(AAm-co-AN) micelles in water at 4,42 , and $70^{\circ} \mathrm{C}$. Reproduced with the permission from reference [61].
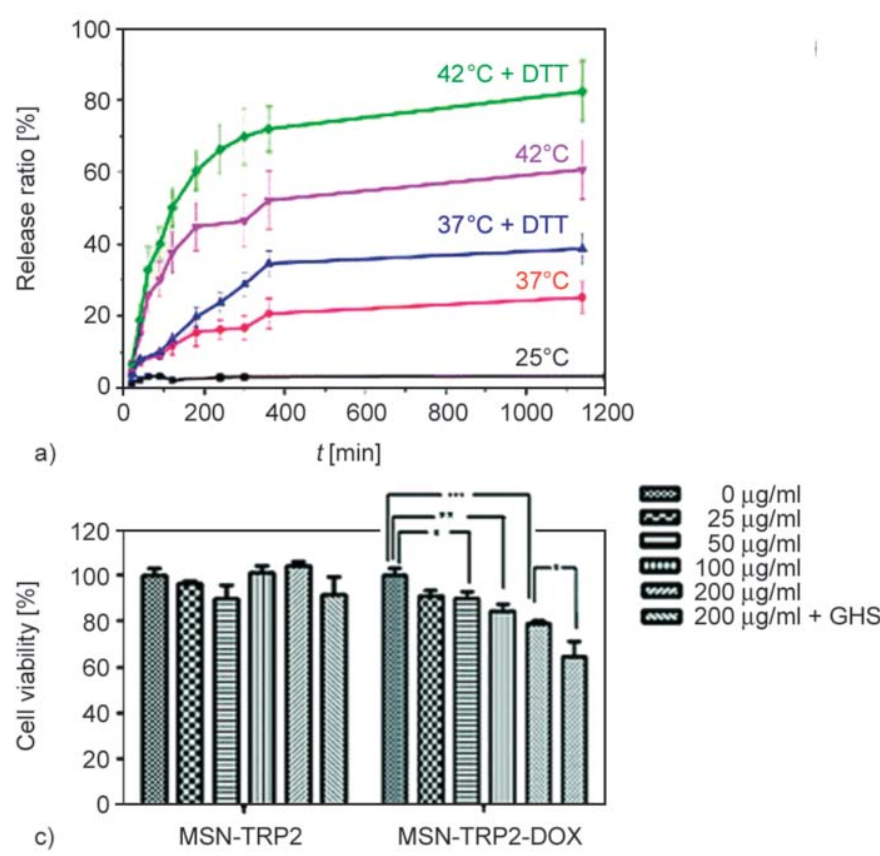

efforts are desirable towards designing a UCST based drug nano-carrier in such fashion that the UCST will be unaffected by drug loading, polymer concentration or by other environmental conditions such as $\mathrm{pH}$ and presence of electrolytes.

A dual responsive drug delivery carrier using P(AAmco-AN) as an UCST block has been developed and evaluated for on-demand release of loaded content (Nile red dye). The triblock copolymer, poly[(ethylene glycol)methyl ether]-block-poly(acrylamide-coacrylonitrile)-block-poly $(N, N$-diethylamino ethyl methacrylate) (PEG- $b$-P(AAm-co-AN)- $b$-PDEAEMA), was synthesized via RAFT polymerization in which PDEAEMA is a $\mathrm{CO}_{2}$-responsive block. Interestingly, this polymer displayed UCST behavior only in the presence of $\mathrm{CO}_{2}$ as it was suggested that the UCST property of $\mathrm{P}(\mathrm{AAm}-\mathrm{co}-\mathrm{AN})$ was diminished due to the attachment of a hydrophobic PDEAEMA block [62]. Similarly, P(AAm-co-AN) copolymer with UCST $\sim 42{ }^{\circ} \mathrm{C}$ was grafted onto mesoporous silica nanoparticles (MSNs) via disulfide linkages, which acted as a gate and offered dual responsive (thermal and redox) controlled release of DOX from the MSN pores. Intracellular release of DOX in SKBR-3 breast cancer cells was controlled successfully by varying temperature and by addition of reducing agent (DTT) as evident by in-vitro experiments
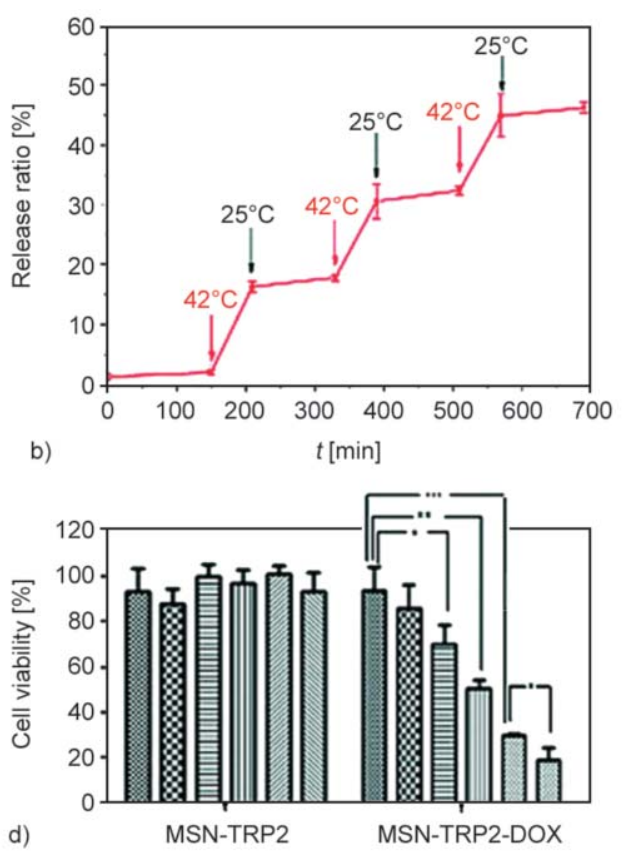

Figure 16. In-vitro DOX release profiles and cell toxicity of MSN-S-S-P(AAm-co-AN) (MSN-TRP2). (a) Drug release from modified MSNs in PBS at $25,37^{\circ} \mathrm{C}, 37^{\circ} \mathrm{C}$ with DTT, $42^{\circ} \mathrm{C}$ and $42^{\circ} \mathrm{C}$ with DTT. (b) Temperature regulated OnOFF release of drug from MSNs above $\left(42^{\circ} \mathrm{C}\right)$ and below UCST $\left(25^{\circ} \mathrm{C}\right)$. Cytotoxicity of MSN-TRP2 and DOX loaded MSN (MSN-TRP2-DOX) to SK-BR-3 cells (c) at $37^{\circ} \mathrm{C}$ and (d) at $42^{\circ} \mathrm{C}$. Reproduced with permission from reference [63]. 
(Figure 16) [63]. Similarly, UCST polymer PEG-gpoly (AAm-co-AN) and poly( $N$-acryloyl glycine) were also utilized as coating material for magnetic nanoparticles to generate multiresponsive drug delivery carriers for temperature regulated release of cargo [64, 65].

Boustta et al. [19] developed a temperature responsive injectable drug delivery system for loco-regional controlled release of drugs using PNAGA polymer, which display gel-sol transition property in physiological medium. Saline solution of PNAGA was prepared by heating above UCST i.e. $\sim 50^{\circ} \mathrm{C}$ wherein hydrophilic model drugs were dissolved, before cooling down below UCST to acquire a gel. These gel formulations demonstrated sustained release of model drugs at physiological temperature, which was also supported by in-vivo studies. It was proposed that injecting this thermoresponsive carrier system in sol form (above UCST) would turn into gel when reaching below UCST i.e. $\sim 37^{\circ} \mathrm{C}$ (human body temperature) and provide sustained release of loaded content.

Deng et al. [66] designed UCST based thermoresponsive polymeric micelles loaded with photothermal agent (IR780) and DOX for addressing the drug resistance issue in cancer therapy. Poly(ethylene glycol)- $b$-poly ( $N$-acryloylglycinamide- $c o$-acrylonitrile) (PEG- $b$-P(NAGA-co-AN)) having $T_{\mathrm{pt}}$ of $44^{\circ} \mathrm{C}$ was utilized to form micelles at room temperature, which disassembled upon NIR irradiation due to increase in temperature above UCST (caused by photothermal effect of IR780), resulting in complete release of DOX (Figure 17). It was suggested that by using UCST based thermoresponsive micelles, a spatial and temporal control release of DOX can be achieved. In addition, synergistic effects in reversing drug resistance in cancer therapy was observed due to IR780-based hyperthermia and DOX-based chemotherapy in drug resistant $\mathrm{MCF}-7$ cells.

Hui et al. [67] utilized copolymer of $N$-acryloylglycinamide (NAGA) and butyl acrylate (BA) (i.e., poly (NAGA-co-BA)) for 'on demand' release of DOX using indocyanine green (ICG) as photothermal agent. The micelles of poly(NAGA-co-BA) (UCST of $\sim 47^{\circ} \mathrm{C}$ and $\mathrm{CMC} \sim 66 \mu \mathrm{g} / \mathrm{ml}$ ) loaded with DOX and ICG was coated with red blood cell as an alternative to PEG to reduce the drug leakage. In a similar fashion, a dual responsiveness (thermo and $\mathrm{pH}$ ) copolymer poly(ethylene glycol)- $b$-poly(acrylamide$c o$-acrylonitrile-co-vinylimidazole) (mPEG-PAAV)

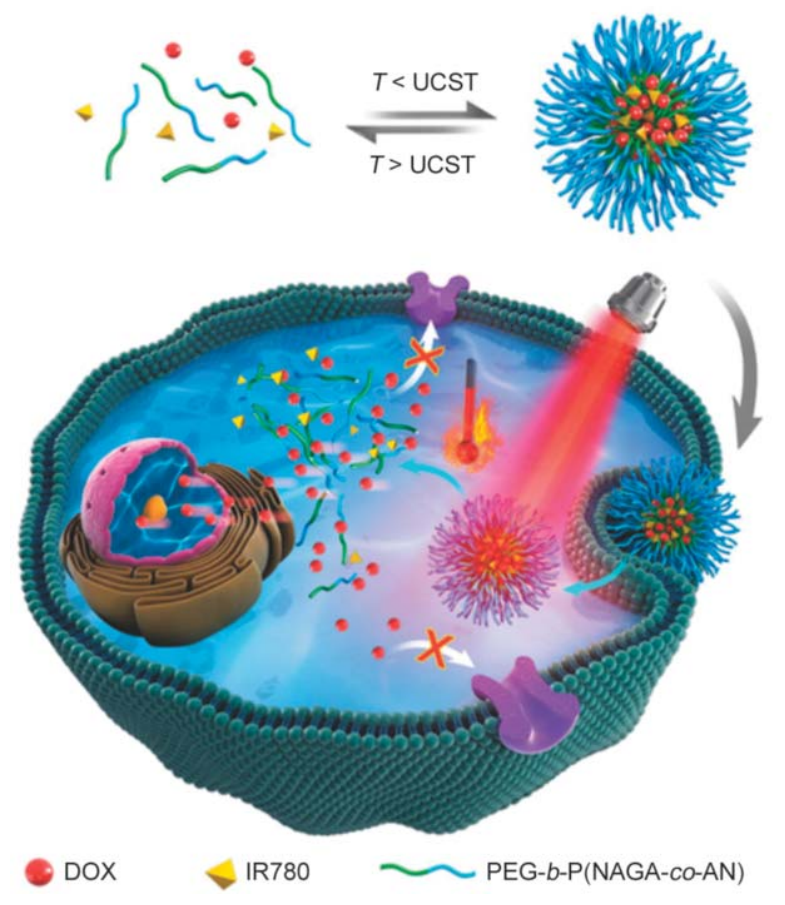

Figure 17. Graphical representation of assembly and disassembly of copolymer PEG- $b$-P(NAGA-co-AN) below and above UCST. NIR light triggered the disassembly of micelles due to increase in temperature inside doxorubicin (DOX) resistant cancer cell. Reproduced with permission from reference [66]. Copyright (C) 2018 WILEY-VCH Verlag GmbH \& Co. KGaA.

was utilized for chemo-photothermal therapy in cancer using DOX and IR780. The UCST of mPEGPAAV micelles at $\mathrm{pH} 7.4$ was $50.8^{\circ} \mathrm{C}$, which decreased significantly when the $\mathrm{pH}$ decreases (45.7, 45.6 and $33.7^{\circ} \mathrm{C}$ at $\mathrm{pH} 6.8,6.5$ and 5.0, respective1y). Since, hydrophobicity played an important role in tuning UCST of the polymer and thus decrease in UCST with $\mathrm{pH}$ was attributed to the presence of imidazole groups, which protonated at lower $\mathrm{pH}$ and increase the total hydrophilicity of the copolymer. Therefore, an accelerated release of DOX was observed at $43^{\circ} \mathrm{C}$ with $\mathrm{pH} 5.0$ (polymer UCST $33.7^{\circ} \mathrm{C}$ ) due to micelles disassembly owing to the temperature above UCST of copolymer [68].

Vesicles of thermoresponsive multi-l-arginyl-poly1-aspartic acid (MAPA) were also employed to control the release rate of DOX [69]. MAPA is a biocompatible nonribosomal polypeptide, which demonstrate thermoresponsive behavior depending on the contents of lysine and arginine [70,71]. A water insoluble multi-L-arginyl-poly-L-aspartate (iMAPA, by keeping lysine content low) was prepared from recombinant Escherichia coli. Later, PEG was attached to the iMAPA ( $70 \%$ to primary 


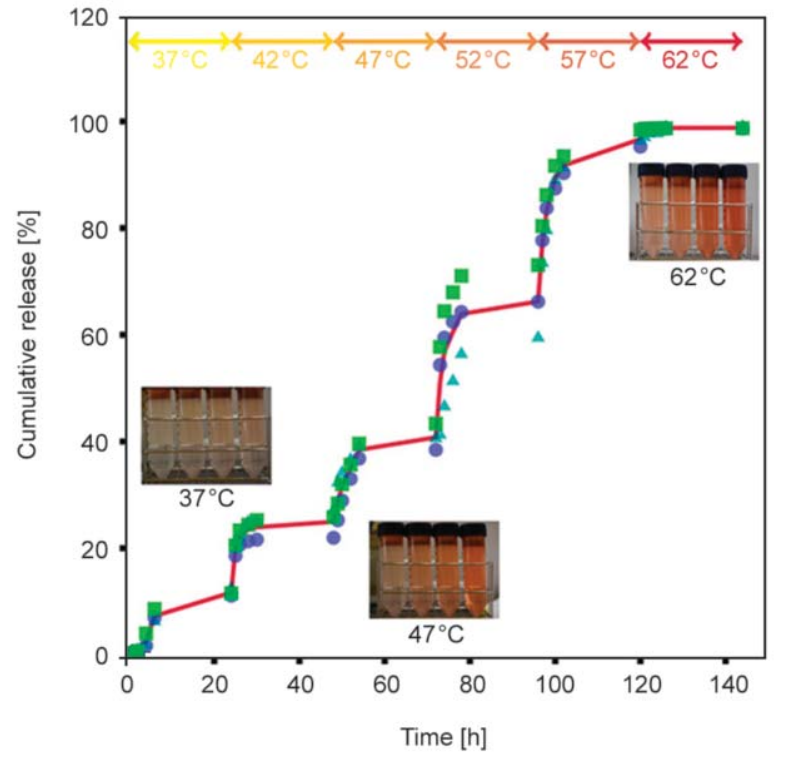

Figure 18. DOX release profile in PBS from vesicles with temperature increase from 37 to $62^{\circ} \mathrm{C}$. Reproduced with permission from [69], Copyright (C) 2018 American Chemical Society.

amine of lysine was substituted) to increase the solubility in physiological medium and to impart UCST behavior. DOX was loaded to this UCST type vesicles, which demonstrated temperature-triggered release in physiological medium (Figure 18) [69]. Ureido based UCST copolymer polyvinylpyrrolidone$b$-polyureido(ornithine-co-lysine) (PVP- $b$-PUOL) have also been investigated for temperature controlled release of loaded molecules [50].

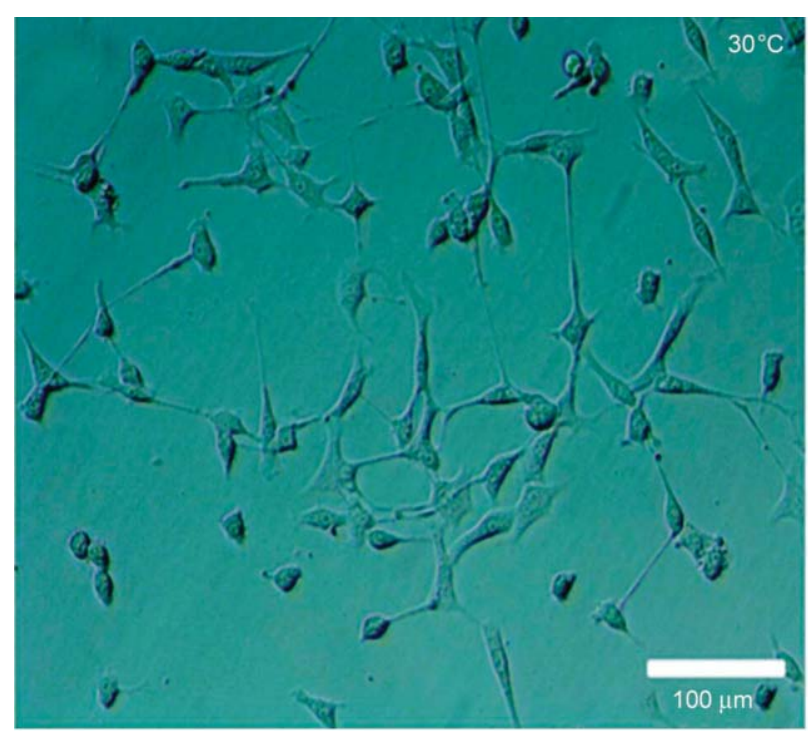

a)

\subsection{Functional cell cultures}

UCST polymers PAU that have pendant ureido groups were investigated for temperature-controlled reversible spheroid/monolayer cell culture switching. NIH-3T3 cells in monolayer culture were switched into spheroids by the addition of PAU polymers below $T_{\mathrm{pt}}\left(\mathrm{UCST} \sim 45^{\circ} \mathrm{C}\right.$ ), which upon heating above $T_{\mathrm{pt}}$, turned back into a monolayer of cells. These results indicated that cell morphology could be repeatedly switched by changing the culture temperature in the presence of ureido polymers. This phenomenon was ascribed to the solubility change of polymer, which separates into two liquid phases below $T_{\mathrm{pt}}$. It was suggested that cell-cell and cell-matrix interactions can be controlled by using these ureido polymers and they could be employed in preparation of cell therapy or tissue engineering devices [72]. In an another study, brushes of UCST-type poly ( $N$-acryloyl glycinamide)-co-poly( $N$-phenylacrylamide) (PNAGAm$\mathrm{PNPhAm}$ ) were prepared for temperature controlled NIH-3T3 cells attachment and detachment. It was observed that cells were attached on the precipitated PNAGAm-PNPhAm brush surface after $20 \mathrm{~h}$ of incubation at $30^{\circ} \mathrm{C}$ (below UCST), whereas cells were detached from the surface within $2 \mathrm{~h}$ when the temperature of culture was increased and maintained at $37^{\circ} \mathrm{C}$ (Figure 19) [73]. $N$-succinimidyl $N$-methylcarbamate modified poly(1-ornithine)-co-poly(1-citrulline) copolymer with UCST of $\sim 39^{\circ} \mathrm{C}$ was also evaluated

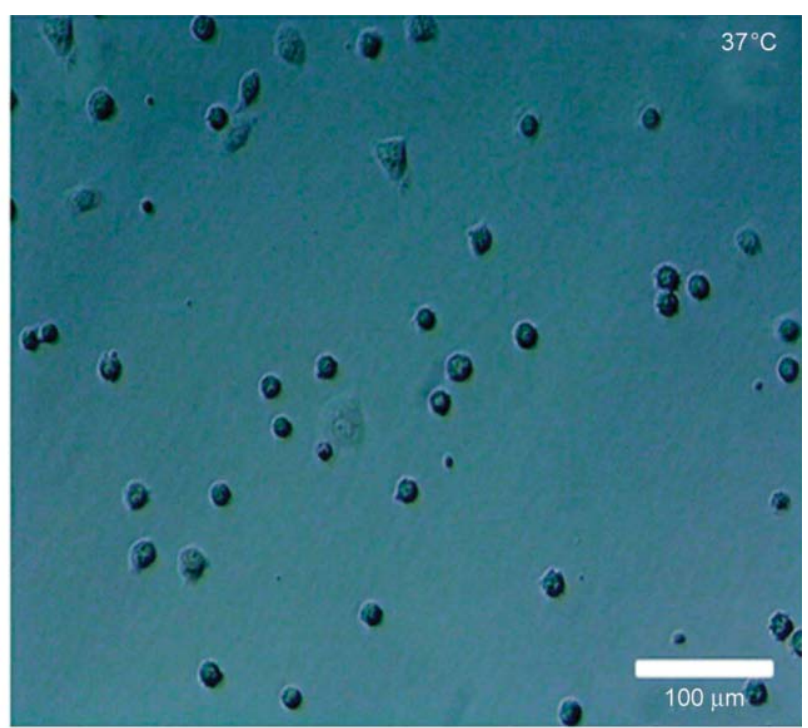

b)

Figure 19. Phase contrast microscopy images of NIH-3T3 cells (a) attached to the polymer brush surface at $30^{\circ} \mathrm{C}$ and (b) detached from surface at $37^{\circ} \mathrm{C}$. Reproduced with permission from reference [73], Copyright (C) 2017, The Royal Society of Chemistry. 
for temperature mediated cell adhesion and detachment. Unlike the previous study, this study demonstrated cell attachment at temperature near UCST while the detachment of cells was observed on reducing the temperature to $37^{\circ} \mathrm{C}$ [74]. Hydrogels are also known to enhance the cells adhesion onto the surface and therefore a thermoresponsive hydrogels based on copolymer of SBE and CBA functionalized with RGD-based peptide has been explored to enhance the 3T3 fibrolast cells attachment onto the plate surface [27].

\subsection{Protein separation}

Thermal denaturation of proteins during bioseperation can be minimized by using UCST polymers since they show phase separation upon cooling. UCST polymer poly(allylurea-co-allylamine) (PUAm, $\left.T_{\mathrm{pt}}-34^{\circ} \mathrm{C}\right)$ and its derivatives i.e. poly(allylurea-co-allylamine) succinate (modified by succinic anhydride, $\mathrm{PU}-\mathrm{Su}, T_{\mathrm{pt}}-16^{\circ} \mathrm{C}$ ) and poly(allylureaco-allylamine) acetate (modified by acetic anhydride, PU-Ac, $T_{\mathrm{pt}}-46^{\circ} \mathrm{C}$ ) have been utilized for protein separation. It was found that PU-Am selectively captured $\beta$-galactosidase, phosphorylase $b$ and soybean trypsin inhibitor (acidic proteins) but was unsuccessful in capturing other acidic proteins such as bovine serum albumin, ovalbumin and carbonic anhydrase. PU-Su selectively captured bovine pancreas trypsin inhibitor and lysozyme (basic proteins); however, PU-Ac did not show notable affinity towards capturing the tested proteins. It was anticipated that electrostatic interactions between ionic groups of proteins and functional groups of polymers might be the main mechanism behind capturing the proteins [20].

\subsection{Improvement in catalytic activity}

Thermosensitive microgels containing silver nanoparticles were prepared to control the catalytic activity of silver. PNAGA was used to prepare microgels at temperatures below UCST by aqueous precipitation polymerization. Upon heating, these microgels swelled in water and exposed silver, which readily catalyse the reduction of 4-nitrophenol in aqueous dispersion. In contrast, at temperatures below UCST, the catalytic activity of silver diminishes, suggesting that temperature regulates an ON-OFF behavior of silver to catalyse reduction reaction with the aid of PNAGA microgels [75]. In an another study, trypsin, a protease enzyme was conjugated with UCST polymer poly(3-dimethyl(methacryloyloxyethyl) ammonium propane sulfonate) (PDMAPS) with the objective of enhancing the stability and activity of the enzyme. As compared to the native trypsin, the conjugate showed a significant enhancement in the thermal stability, particularly at high temperatures. Hydrolysis of casein (a high molecular weight substrate) using native trypsin and its conjugate was tested at $60^{\circ} \mathrm{C}$ and found that trypsin activity was increased by $2.6-$ fold in conjugate form, whereas $48 \%$ decrease in activity was observed with native trypsin. It is also worth noting that the conjugate can be easily recovered and reused for at least 10 more cycles without losing the original activity (only $15 \%$ reduction in activity was observed) by simply reducing the temperature below UCST [21].

Similarly, enzyme Pseudomonas Cepacia Lipase (PSL) was attached to copolymer P(AAm-co-AN) in order to enhance the catalytic performance and stability of the enzyme on a broader $\mathrm{pH}$ and temperature range. It was observed that the conjugated PSL maintained $68.8 \%$ of its original activity after $12 \mathrm{~h}$ of incubation at $60^{\circ} \mathrm{C}$, whereas free PSL retained only $34.4 \%$ of its original activity. Facile recovery of this biocatalyst was also demonstrated below $\operatorname{UCST}\left(0^{\circ} \mathrm{C}\right)$ and it was reused up to six cycles with retainment of $80.5 \%$ of its original activity [76]. Hydrolysis of cellulose to glucose using cellulase enzyme is always a troublesome process due to poor accessibility of immobilized cellulase to insoluble cellulose, and difficulty in recovery of immobilized enzyme from remaining insoluble substrate. To solve this problem, poly(methacrylamide-co-acrylic acid) (PMAAc) with UCST of $16^{\circ} \mathrm{C}$ was utilized to immobilized cellulase enzyme in order to enhance the stability at high temperature and to enable easy recovery of enzyme below UCST. The hydrolysis rate of insoluble cellulose to glucose at elevated temperature was significantly enhanced with the aid of newly developed biocatalyst while retaining the enzyme activity [77].

\subsection{Sensors}

Temperature responsive UCST materials can be used as sensors in various applications that upon temperature change could raise the alarm. In this sequence, Danko et al. [27] prepared thermoresponsive hydrogel of SBE and CBA using ethylene glycol dimethacrylate. This hydrogel turns transparent above UCST and can be used as a visible indicator for cooling needs (Figure 20). The warning temperature can be 

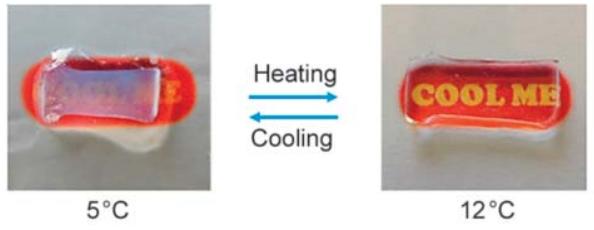

Figure 20. Representative image giving the visible warning for cooling when the temperature rise from 5 to $12{ }^{\circ} \mathrm{C}$. Reproduced with permission from [27], Copyright (C) 2019 American Chemical Society.

easily tuned as per the need by changing the content of SBE in CBA.

\section{Conclusions}

This review article summarized the synthesis approaches and applications of various polymers exhibiting upper critical solution temperature. The UCST polymers can be classified into two categories, i.e. polymer showing UCST based on hydrogen bonding and those based on electrostatic interactions. The UCST of polymers can be easily tuned by changing the constructs of the hydrophobic moiety in the polymer, by varying the molecular weight or by synthesizing copolymers. Although sufficient efforts have been made to synthesize novel polymers with tunable UCST, their applications are sparsely explored. It is apparent from the application part of this article that the most investigated application field for UCST polymers is drug delivery, specifically controlled release. Thus, in this particular field, polymers showing UCST via hydrogen bonding are more advantageous since their UCST does not affect by the presence of electrolytes/salts in solvent. Moreover, UCST dependent on polymer concentration in solution is certainly a serious flaw limiting the applications of these carriers in drug delivery, due to the fact that formulations are subjected to getting diluted by blood after IV administration. In addition, limited information is available on stability and toxicity profiles of these polymeric systems. Therefore, future studies should be more focused towards making biodegradable and biocompatible polymers with stable UCST in order to attain reproducibility. More specifically, designing polymers with UCST independent to polymer concentration, $\mathrm{pH}$, electrolytes and so forth are of great interest. Further, to establish the practicality of these polymers, additional efforts are highly desirable to unravel novel applications in supplemental areas.

\section{Acknowledgements}

This work was supported by The Ella and Georg Ehrnrooth Foundation grant to K.K.B. and by the Academy of Finland (project \#309374).

\section{References}

[1] Guragain S., Bastakoti B. P., Malgras V., Nakashima K., Yamauchi Y.: Multi-stimuli-responsive polymeric materials. Chemistry - A European Journal, 21, 13164 13174 (2015). https://doi.org/10.1002/chem.201501101

[2] Gandhi A., Paul A., Sen S. O., Sen K. K.: Studies on thermoresponsive polymers: Phase behaviour, drug delivery and biomedical applications. Asian Journal of Pharmaceutical Sciences, 10, 99-107 (2015). https://doi.org/10.1016/j.ajps.2014.08.010

[3] Li Q., Li L., Tian Q., Xu J., Liu J.: Doubly thermo-responsive polymers and their two-step phase transition behavior: A review. Nanoscience and Nanotechnology Letters, 9, 89-99 (2017). https://doi.org/10.1166/nnl.2017.2266

[4] Käfer F., Liu F., Stahlschmidt U., Jérôme V., Freitag R., Karg M., Agarwal S.: LCST and UCST in one: Double thermoresponsive behavior of block copolymers of poly(ethylene glycol) and poly(acrylamide-co-acrylonitrile). Langmuir, 31, 8940-8946 (2015).

https://doi.org/10.1021/acs.langmuir.5b02006

[5] Zhang Q., Hoogenboom R.: Polymers with upper critical solution temperature behavior in alcohol/water solvent mixtures. Progress in Polymer Science, 48, 122142 (2015). https://doi.org/10.1016/j.progpolymsci.2015.02.003

[6] Priya James H., John R., Alex A., Anoop K. R.: Smart polymers for the controlled delivery of drugs - A concise overview. Acta Pharmaceutica Sinica B, 4, 120127 (2014). https://doi.org/10.1016/j.apsb.2014.02.005

[7] Zhu Y., Batchelor R., Lowe A. B., Roth P. J.: Design of thermoresponsive polymers with aqueous LCST, UCST, or both: Modification of a reactive poly(2-vinyl-4,4-dimethylazlactone) scaffold. Macromolecules, 49, 672680 (2016).

https://doi.org/10.1021/acs.macromol.5b02056

[8] Niskanen J., Tenhu H.: How to manipulate the upper critical solution temperature (UCST)? Polymer Chemistry, 8, 220-232 (2017).

https://doi.org/10.1039/C6PY01612J

[9] Seuring J., Agarwal S.: Polymers with upper critical solution temperature in aqueous solution. Macromolecular Rapid Communications, 33, 1898-1920 (2012). https://doi.org/10.1002/marc.201200433

[10] Seuring J., Agarwal S.: Non-ionic homo- and copolymers with $\mathrm{H}$-donor and $\mathrm{H}$-acceptor units with an UCST in water. Macromolecular Chemistry and Physics, 211, 2109-2117 (2010). https://doi.org/10.1002/macp.201000147 
[11] Teotia A. K., Sami H., Kumar A.: Thermo-responsive polymers: Structure and design of smart materials. in 'Switchable and responsive surfaces and materials for biomedical applications' (ed.: Zhang Z.) Woodhead, Oxford, 3-43 (2015).

https://doi.org/10.1016/B978-0-85709-713-2.00001-8

[12] Clark E. A., Lipson J. E. G.: LCST and UCST behavior in polymer solutions and blends. Polymer, 53, 536-545 (2012).

https://doi.org/10.1016/j.polymer.2011.11.045

[13] Qiu Y., Park K.: Environment-sensitive hydrogels for drug delivery. Advanced Drug Delivery Reviews, 64, 49-60 (2012).

https://doi.org/10.1016/j.addr.2012.09.024

[14] Kim Y-J., Matsunaga Y. T.: Thermo-responsive polymers and their application as smart biomaterials. Journal of Materials Chemistry B, 5, 4307-4321 (2017). https://doi.org/10.1039/C7TB00157F

[15] Jochum F. D., Theato P.: Temperature- and light-responsive smart polymer materials. Chemical Society Reviews, 42, 7468-7483 (2013). https://doi.org/10.1039/C2CS35191A

[16] Roy D., Brooks W. L. A., Sumerlin B. S.: New directions in thermoresponsive polymers. Chemical Society Reviews, 42, 7214-7243 (2013).

https://doi.org/10.1039/C3CS35499G

[17] Shimada N., Maruyama A.: Thermoresponsive polymers with functional groups selected for pharmaceutical and biomedical applications. in 'Tailored polymer architectures for pharmaceutical and biomedical applications' (eds.: American Chemical Society, 1135, 235 241 (2013).

https://doi.org/10.1021/bk-2013-1135.ch014

[18] Chen L., Honma Y., Mizutani T., Liaw D-J., Gong J. P., Osada Y.: Effects of polyelectrolyte complexation on the UCST of zwitterionic polymer. Polymer, 41, 141147 (2000).

https://doi.org/10.1016/S0032-3861(99)00161-5

[19] Boustta M., Colombo P-E., Lenglet S., Poujol S., Vert M.: Versatile UCST-based thermoresponsive hydrogels for loco-regional sustained drug delivery. Journal of Controlled Release, 174, 1-6 (2014).

https://doi.org/10.1016/j.jconrel.2013.10.040

[20] Shimada N., Nakayama M., Kano A., Maruyama A.: Design of UCST polymers for chilling capture of proteins. Biomacromolecules, 14, 1452-1457 (2013). https://doi.org/10.1021/bm400120y

[21] Yan M., Ge J., Dong W., Liu Z., Ouyang P.: Preparation and characterization of a temperature-sensitive sulfobetaine polymer-trypsin conjugate. Biochemical Engineering Journal, 30, 48-54 (2006).

https://doi.org/10.1016/j.bej.2006.02.001

[22] Zhu Y., Noy J-M., Lowe A. B., Roth P. J.: The synthesis and aqueous solution properties of sulfobutylbetaine (co)polymers: Comparison of synthetic routes and tuneable upper critical solution temperatures. Polymer Chemistry, 6, 5705-5718 (2015).

https://doi.org/10.1039/C5PY00160A
[23] Lim J., Matsuoka H., Yusa S-I., Saruwatari Y.: Temperature-responsive behavior of double hydrophilic carboxy-sulfobetaine block copolymers and their self-assemblies in water. Langmuir, 35, 1571-1582 (2019). https://doi.org/10.1021/acs.langmuir.8b02952

[24] Mary P., Bendejacq D. D., Labeau M-P., Dupuis P.: Reconciling low- and high-salt solution behavior of sulfobetaine polyzwitterions. The Journal of Physical Chemistry B, 111, 7767-7777 (2007).

https://doi.org/10.1021/jp071995b

[25] Woodfield P. A., Zhu Y., Pei Y., Roth P. J.: Hydrophobically modified sulfobetaine copolymers with tunable aqueous UCST through postpolymerization modification of poly(pentafluorophenyl acrylate). Macromolecules, 47, 750-762 (2014).

https://doi.org/10.1021/ma402391a

[26] Hildebrand V., Laschewsky A., Päch M., MüllerBuschbaum P., Papadakis C. M.: Effect of the zwitterion structure on the thermo-responsive behaviour of poly(sulfobetaine methacrylates). Polymer Chemistry, 8, 310-322 (2017). https://doi.org/10.1039/C6PY01220E

[27] Danko M., Kroneková Z., Mrlik M., Osicka J., bin Yousaf A., Mihálová A., Tkac J., Kasak P.: Sulfobetaines meet carboxybetaines: Modulation of thermoand ion-responsivity, water structure, mechanical properties, and cell adhesion. Langmuir, 35, 1391-1403 (2019).

https://doi.org/10.1021/acs.langmuir.8b01592

[28] Vishnevetskaya N. S., Hildebrand V., Niebuur B-J., Grillo I., Filippov S. K., Laschewsky A., MüllerBuschbaum P., Papadakis C. M.: 'Schizophrenic' micelles from doubly thermoresponsive polysulfobetaine$b$-poly $(N$-isopropylmethacrylamide) diblock copolymers. Macromolecules, 50, 3985-3999 (2017).

https://doi.org/10.1021/acs.macromol.7b00356

[29] Doncom K. E. B., Willcock H., O’Reilly R. K.: The direct synthesis of sulfobetaine-containing amphiphilic block copolymers and their self-assembly behavior. European Polymer Journal, 87, 497-507 (2017). https://doi.org/10.1016/j.eurpolymj.2016.09.002

[30] Nizardo N. M., Schanzenbach D., Schönemann E., Laschewsky A.: Exploring poly(ethylene glycol)-polyzwitterion diblock copolymers as biocompatible smart macrosurfactants featuring UCST-phase behavior in normal saline solution. Polymers, 10, 325/1-325/22 (2018).

https://doi.org/10.3390/polym10030325

[31] Christensen L. H., Breiting V. B., Aasted A., Jørgensen A., Kebuladze I.: Long-term effects of polyacrylamide hydrogel on human breast tissue. Plastic and reconstructive surgery, 111, 1883-1890 (2003). https://doi.org/10.1097/01.prs.0000056873.87165.5a

[32] Patrick T.: Polyacrylamide gel in cosmetic procedures: Experience with aquamid. Seminars in Cutaneous Medicine and Surgery, 23, 233-235 (2004). https://doi.org/10.1016/j.sder.2004.09.003 
[33] Seuring J., Agarwal S.: First example of a universal and cost-effective approach: Polymers with tunable upper critical solution temperature in water and electrolyte solution. Macromolecules, 45, 3910-3918 (2012). https://doi.org/10.1021/ma300355k

[34] Asadujjaman A., Kent B., Bertin A.: Phase transition and aggregation behaviour of an UCST-type copolymer poly(acrylamide-co-acrylonitrile) in water: Effect of acrylonitrile content, concentration in solution, copolymer chain length and presence of electrolyte. Soft Matter, 13, 658-669 (2017). https://doi.org/10.1039/C6SM02262F

[35] Hou L., Wu P.: Understanding the UCST-type transition of $\mathrm{P}(\mathrm{AAm}-\mathrm{co}-\mathrm{AN})$ in $\mathrm{H}_{2} \mathrm{O}$ and $\mathrm{D}_{2} \mathrm{O}$ : Dramatic effects of solvent isotopes. Soft Matter, 11, 7059-7065 (2015). https://doi.org/10.1039/C5SM01745A

[36] Jia Y-G., Yu Q., Ma Z., Zhang M., Zhu X. X.: Tunable upper critical solution temperatures for acrylamide copolymers with bile acid pendants. Biomacromolecules, 18, 2663-2668 (2017).

https://doi.org/10.1021/acs.biomac.7b00860

[37] Zhang H., Tong X., Zhao Y.: Diverse thermoresponsive behaviors of uncharged UCST block copolymer micelles in physiological medium. Langmuir, 30, 11433 11441 (2014). https://doi.org/10.1021/la5026334

[38] Palanisamy A., Sukhishvili S. A.: Swelling transitions in layer-by-layer assemblies of UCST block copolymer micelles. Macromolecules, 51, 3467-3476 (2018). https://doi.org/10.1021/acs.macromol.8b00519

[39] Qi M., Li K., Zheng Y., Rasheed T., Zhou Y.: Hyperbranched multiarm copolymers with a UCST phase transition: Topological effect and the mechanism. Langmuir, 34, 3058-3067 (2018).

https://doi.org/10.1021/acs.langmuir.7b04255

[40] Fu W., Zhao B.: Thermoreversible physically crosslinked hydrogels from UCST-type thermosensitive ABA linear triblock copolymers. Polymer Chemistry, 7, 6980-6991 (2016). https://doi.org/10.1039/C6PY01517D

[41] Augé A., Fortin D., Tong X., Zhao Y.: Nanogel-like UCST triblock copolymer micelles showing large volume expansion before abrupt dissolution. Polymer Chemistry, 9, 4660-4673 (2018). https://doi.org/10.1039/C8PY00960K

[42] Seuring J., Bayer F. M., Huber K., Agarwal S.: Upper critical solution temperature of $\operatorname{poly}(n$-acryloyl glycinamide) in water: A concealed property. Macromolecules, 45, 374-384 (2012).

https://doi.org/10.1021/ma202059t

[43] Xu Z., Liu W.: Poly(n-acryloyl glycinamide): A fascinating polymer that exhibits a range of properties from UCST to high-strength hydrogels. Chemical Communications, 54, 10540-10553 (2018).

https://doi.org/10.1039/C8CC04614J
[44] Käfer F., Lerch A., Agarwal S.: Tunable, concentrationindependent, sharp, hysteresis-free UCST phase transition from poly( $\mathrm{N}$-acryloyl glycinamide-acrylonitrile) system. Journal of Polymer Science Part A: Polymer Chemistry, 55, 274-279 (2017).

https://doi.org/10.1002/pola.28374

[45] Sun W., An Z., Wu P.: UCST or LCST? Compositiondependent thermoresponsive behavior of poly $(n$-acryloylglycinamide-co-diacetone acrylamide). Macromolecules, 50, 2175-2182 (2017).

https://doi.org/10.1021/acs.macromol.7b00020

[46] Sun W., An Z., Wu P.: Hydrogen bonding reinforcement as a strategy to improve upper critical solution temperature of $\operatorname{poly}(n$-acryloylglycinamide-co-methacrylic acid). Polymer Chemistry, 9, 3667-3673 (2018).

https://doi.org/10.1039/C8PY00733K

[47] Shimada N., Ino H., Maie K., Nakayama M., Kano A., Maruyama A.: Ureido-derivatized polymers based on both poly(allylurea) and poly(l-citrulline) exhibit UCST-type phase transition behavior under physiologically relevant conditions. Biomacromolecules, 12, 3418-3422 (2011).

https://doi.org/10.1021/bm2010752

[48] Shimada N., Sasaki T., Kawano T., Maruyama A.: Rational design of UCST-type ureido copolymers based on a hydrophobic parameter. Biomacromolecules, 19, 4133-4138 (2018). https://doi.org/10.1021/acs.biomac.8b01152

[49] Kuroyanagi S., Shimada N., Fujii S., Furuta T., Harada A., Sakurai K., Maruyama A.: Highly ordered polypeptide with UCST phase separation behavior. Journal of the American Chemical Society, 141, 1261-1268 (2019). https://doi.org/10.1021/jacs.8b10168

[50] Palanisamy A., Albright V., Sukhishvili S. A.: Upper critical solution temperature layer-by-layer films of polyamino acid-based micelles with rapid, on-demand release capability. Chemistry of Materials, 29, 90849094 (2017). https://doi.org/10.1021/acs.chemmater.7b02748

[51] Zhou Q., Palanisamy A., Albright V., Sukhishvili S. A.: Enzymatically degradable star polypeptides with tunable UCST transitions in solution and within layer-bylayer films. Polymer Chemistry, 9, 4979-4983 (2018). https://doi.org/10.1039/C8PY00939B

[52] Fujihara A., Itsuki K., Shimada N., Maruyama A., Sagawa N., Shikata T., Yusa S-I.: Preparation of ureido group bearing polymers and their upper critical solution temperature in water. Journal of Polymer Science Part A: Polymer Chemistry, 54, 2845-2854 (2016). https://doi.org/10.1002/pola.28183

[53] Fujihara A., Shimada N., Maruyama A., Ishihara K., Nakai K., Yusa S-I.: Preparation of upper critical solution temperature (UCST) responsive diblock copolymers bearing pendant ureido groups and their micelle formation behavior in water. Soft Matter, 11, 52045213 (2015).

https://doi.org/10.1039/C5SM00499C 
[54] Wolf T., Rheinberger T., Simon J., Wurm F. R.: Reversible self-assembly of degradable polymersomes with upper critical solution temperature in water. Journal of the American Chemical Society, 139, 11064 11072 (2017). https://doi.org/10.1021/jacs.7b02723

[55] Di Y., Ma X., Li C., Liu H., Fan X., Wang M., Deng H., Jiang T., Yin Z., Deng K.: A new thermosensitive poly ( $N$-propionyl-aspartic acid/ethylene glycol) with no cytotoxicity and tunable UCST. Macromolecular Chemistry and Physics, 215, 365-371 (2014). https://doi.org/10.1002/macp.201300687

[56] Ye H., Owh C., Loh X. J.: A thixotropic polyglycerol sebacate-based supramolecular hydrogel showing UCST behavior. RSC Advances, 5, 48720-48728 (2015). https://doi.org/10.1039/C5RA08222F

[57] Wu G., Chen S-C., Zhan Q., Wang Y-Z.: Well-defined amphiphilic biodegradable comb-like graft copolymers: Their unique architecture-determined LCST and UCST thermoresponsivity. Macromolecules, 44, 999-1008 (2011). https://doi.org/10.1021/ma102588k

[58] Zhang G., Lei J., Wu L., Guo C., Fang J., Bai R., Wyman I.: Poly(imidazoled glycidyl methacrylate-co-diethyleneglycol methyl ether methacrylate) - A new copolymer with tunable LCST and UCST behavior in water. Polymer, 157, 79-86 (2018).

https://doi.org/10.1016/j.polymer.2018.10.026

[59] Shen J., Han K., Martin E. J., Wu Y. Y., Kung M. C., Hayner C. M., Shull K. R., Kung H. H.: Upper-critical solution temperature (UCST) polymer functionalized graphene oxide as thermally responsive ion permeable membrane for energy storage devices. Journal of Materials Chemistry A, 2, 18204-18207 (2014). https://doi.org/10.1039/C4TA04852K

[60] Li W., Huang L., Ying X., Jian Y., Hong Y., Hu F., Du Y.: Antitumor drug delivery modulated by a polymeric micelle with an upper critical solution temperature. Angewandte Chemie - International Edition, 54, 3126-3131 (2015).

https://doi.org/10.1002/anie.201411524

[61] Huang G., Li H., Feng S-T., Li X., Tong G., Liu J., Quan C., Jiang Q., Zhang C., Li Z.: Self-assembled UCSTtype micelles as potential drug carriers for cancer therapeutics. Macromolecular Chemistry and Physics, 216, 1014-1023 (2015).

https://doi.org/10.1002/macp.201400546

[62] Lin S., Shang J., Theato P.: $\mathrm{Co}_{2}$-triggered UCST transition of amphiphilic triblock copolymers and their selfassemblies. Polymer Chemistry, 8, 2619-2629 (2017). https://doi.org/10.1039/C7PY00186J

[63] Hei M., Wang J., Wang K., Zhu W., Ma P. X.: Dually responsive mesoporous silica nanoparticles regulated by upper critical solution temperature polymers for intracellular drug delivery. Journal of Materials Chemistry B, 5, 9497-9501 (2017).

https://doi.org/10.1039/C7TB02429K
[64] Deepuppha N., Khadsai S., Rutnakornpituk B., Wichai U., Rutnakornpituk M.: Multiresponsive poly(n-acryloyl glycine)-based nanocomposite and its drug release characteristics. Journal of Nanomaterials, 2019, 8252036/1-8252036/12 (2019). https://doi.org/10.1155/2019/8252036

[65] Wu L., Zong L., Ni H., Liu X., Wen W., Feng L., Cao J., Qi X., Ge Y., Shen S.: Magnetic thermosensitive micelles with upper critical solution temperature for NIR triggered drug release. Biomaterials Science, 7, 21342143 (2019).

https://doi.org/10.1039/C8BM01672K

[66] Deng Y., Käfer F., Chen T., Jin Q., Ji J., Agarwal S.: Let there be light: Polymeric micelles with upper critical solution temperature as light-triggered heat nanogenerators for combating drug-resistant cancer. Small, 14, 1802420/1-1802420/10 (2018).

https://doi.org/10.1002/smll.201802420

[67] Hui L., Qin S., Yang L.: Upper critical solution temperature polymer, photothermal agent, and erythrocyte membrane coating: An unexplored recipe for making drug carriers with spatiotemporally controlled cargo release. ACS Biomaterials Science and Engineering, 2, 2127-2132 (2016).

https://doi.org/10.1021/acsbiomaterials.6b00459

[68] Yang Z., Cheng R., Zhao C., Sun N., Luo H., Chen Y., Liu Z., Li X., Liu J., Tian Z.: Thermo- and pH-dual responsive polymeric micelles with upper critical solution temperature behavior for photoacoustic imaging-guided synergistic chemo-photothermal therapy against subcutaneous and metastatic breast tumors. Theranostics, 8 , 4097-4115 (2018). https://doi.org/10.7150/thno.26195

[69] Tseng W-C., Fang T-Y., Lin Y-C., Huang S-J., Huang Y-H.: Reversible self-assembly nanovesicle of UCST response prepared with multi-l-arginyl-poly-1-aspartate conjugated with polyethylene glycol. Biomacromolecules, 19, 4585-4592 (2018).

https://doi.org/10.1021/acs.biomac.8b01274

[70] Frommeyer M., Steinbüchel A.: Increased lysine content is the main characteristic of the soluble form of the polyamide cyanophycin synthesized by recombinant Escherichia coli. Applied and Environmental Microbiology, 79, 4474-4483 (2013). https://doi.org/10.1128/aem.00986-13

[71] Tseng W-C., Fang T-Y., Hsieh Y-C., Chen C-Y., Li M-C.: Solubility and thermal response of fractionated cyanophycin prepared with recombinant Escherichia coli. Journal of Biotechnology, 249, 59-65 (2017). https://doi.org/10.1016/j.jbiotec.2017.04.001

[72] Shimada N., Saito M., Shukuri S., Kuroyanagi S., Kuboki T., Kidoaki S., Nagai T., Maruyama A.: Reversible monolayer/spheroid cell culture switching by UCST-type thermoresponsive ureido polymers. ACS Applied Materials \& Interfaces, 8, 31524-31529 (2016). https://doi.org/10.1021/acsami.6b07614 
[73] Xue X., Thiagarajan L., Braim S., Saunders B. R., Shakesheff K. M., Alexander C.: Upper critical solution temperature thermo-responsive polymer brushes and a mechanism for controlled cell attachment. Journal of Materials Chemistry B, 5, 4926-4933 (2017). https://doi.org/10.1039/C7TB00052A

[74] Xue X., Thiagarajan L., Dixon J. E., Saunders B. R., Shakesheff K. M., Alexander C.: Post-modified polypeptides with UCST-type behavior for control of cell attachment in physiological conditions. Materials, 11, 95/1-95/17 (2018). https://doi.org/10.3390/ma11010095

[75] Yang D., Viitasuo M., Pooch F., Tenhu H., Hietala S.: Poly( $N$-acryloylglycinamide) microgels as nanocatalyst platform. Polymer Chemistry, 9, 517-524 (2018). https://doi.org/10.1039/c7py01950e
[76] Lou L-L., Qu H., Yu W., Wang B., Ouyang L., Liu S., Zhou W.: Covalently immobilized lipase on a thermoresponsive polymer with an upper critical solution temperature as an efficient and recyclable asymmetric catalyst in aqueous media. ChemCatChem, 10, 1166-1172 (2018).

https://doi.org/10.1002/cctc.201701512

[77] Han J., Wan J., Wang Y., Wang L., Li C., Mao Y., Ni L.: Recyclable soluble-insoluble upper critical solution temperature-type poly(methacrylamide-co-acrylic acid)cellulase biocatalyst for hydrolysis of cellulose into glucose. ACS Sustainable Chemistry and Engineering, 6, 7779-7788 (2018).

https://doi.org/10.1021/acssuschemeng.8b00769 